\title{
A CURRENT AND VIBRATION BASED DETECTION SYSTEM FOR LIGHTNING STRIKES ON TRANSMISSION TOWERS
}

\author{
A Thesis \\ Presented to \\ The Graduate Faculty of The University of Akron \\ In Partial Fulfillment \\ of the Requirements for the Degree \\ Master of Science
}

Taohid Latif

December 2018 
A CURRENT AND VIBRATION BASED DETECTION SYSTEM FOR

LIGHTNING STRIKES ON TRANSMISSION TOWERS

Taohid Latif

Thesis

Approved:

Accepted:

Advisor

Dr. Nathan Ida

Interim Department Chair

Dr. Robert Veillette

Committee Member

Dr. Kye-Shin Lee

Interim Dean of the College

Dr. Craig Menzemer

Committee Member

Dean of the Graduate School

Dr. Joan Carletta

Dr. Chand K. Midha

Date 


\begin{abstract}
Lightning strikes can lead to disastrous situations in transmission lines and towers and condition monitoring is a crucial step in mitigating the probable adverse effects associated with this natural phenomenon. The current work proposes a method and circuitry for a lightning strike detection system with current and vibration detecting features. Each tower in a network can be equipped with this detection system. The current detector uses a current transformer called Rogowski coil along with additional components in a circuit. Due to its reliance on an air cored coil, the Rogowski coil does not have any issues with magnetization errors and is able to measure and detect ac and fast changing impulse currents. It can be placed around a conductor where the lightning current is expected to flow. Simulation results were used to verify the behavior of the current detector with $300 \mathrm{~mA}$ and $100 \mathrm{kA}$ currents over a frequency range from $1 \mathrm{kHz}$ to $9.57 \mathrm{MHz}$. A small scale prototype was built that performed adequately and the experimental results obtained were in line with the theoretical and simulation results. The detection system also provides means of ascertaining which tower is closest during the moment of the strike through use of a dual-axis digital accelerometer by making use of the vibration at the moment of the strike and correlating that with the current detected. The vibration detector was tested using varying levels of vibration. The current and vibration detector were then unified via a microcontroller to make up the lightning strike detector.
\end{abstract}




\section{ACKNOWLEDGMENTS}

I would like to thank my advisor, Dr. Ida for his guidance, advice and encouragement. I did not have any prior knowledge regarding the world of sensors and without his understanding and immense knowledge regarding the subject matter, I would not have been able to come this far. I really appreciate all that he has done for me and the whole experience of working under his supervision has been instrumental to the completion of my graduate studies. I would also like to thank my thesis committee members, Dr. Lee and Dr. Carletta who were always there whenever I needed any help regarding my research. I would also like to thank Mr. Eric Rinaldo for his assistance on the project.

For the last but, certainly, not the least, I would like to thank my friends and family, especially my parents, brother and sister-in-law for their advice and support. I will always be grateful to them and mere words would not do justice for fully showing my appreciation towards them. 
TABLE OF CONTENTS

Page

LIST OF TABLES vii

LIST OF FIGURES viii

CHAPTER

I. INTRODUCTION 1

1.1 Overview 1

1.2 Objectives of the thesis 4

$\begin{array}{lll}1.3 & \text { Thesis organization } & 7\end{array}$

II. CURRENT DETECTOR 8

2.1 Block diagram of the proposed current detector 8

$\begin{array}{lll}2.2 & \text { Rogowski coil } & 9\end{array}$

$\begin{array}{lll}2.3 & \text { Impedance matching circuit } & 17\end{array}$

$\begin{array}{lll}2.4 & \text { Metal oxide varistor (MOV) } & 17\end{array}$

$\begin{array}{lll}2.5 & \text { Full wave rectifier } & 19\end{array}$

2.6 RC integrator 20

$\begin{array}{lll}2.7 & \text { Proposed current detector } & 20\end{array}$

$\begin{array}{lll}2.8 \text { Summary } & 21\end{array}$

III. VIBRATION DETECTOR 23

3.1 Block diagram of the proposed vibration detector 23

3.2 Accelerometer 24

3.3 MEMSIC 2125 dual-axis accelerometer 27

$\begin{array}{lll}3.4 & \text { Microcontroller } & 29\end{array}$

3.5 Proposed vibration detector 30

$\begin{array}{lll}3.6 & \text { Summary } & 32\end{array}$ 
IV. COMBINED LIGHTNING STRIKE DETECTION SYSTEM

4.1 Lightning strike detector system 33

4.2 Summary 36

V. RESULTS FOR THE LIGHTNING STRIKE DETECTOR 37

5.1 Simulation results for the current detector 37

5.2 Experimental results for the current detector 46

5.3 Results for the vibration detector $\quad 51$

5.4 Results for the combined lightning strike detector 53

$\begin{array}{lll}5.5 & \text { Summary } & 55\end{array}$

VI. CONCLUSION AND FUTURE WORK 56

6.1 Conclusion $\quad 56$

$\begin{array}{ll}6.2 \text { Future work } & 57\end{array}$

$\begin{array}{ll}\text { REFERENCES } & 58\end{array}$

$\begin{array}{ll}\text { APPENDICES } & 62\end{array}$

APPENDIX A: EXPERIMENTAL SETUP 63

APPENDIX B: ARDUINO CODE 64 


\section{LIST OF TABLES}

Table

Page

3.1 Pin description of MEMSIC 2125

28

A.1 List of components

63 


\section{LIST OF FIGURES}

Figure Page

$1.1 \quad$ A tower that failed under broken conductor conditions 1

1.2 A coil acting as the secondary coil of a current transformer 3

1.3 Current propagation during a lightning strike 5

1.4 Shockwave propagation during a lightning strike 6

$2.1 \quad$ Block diagram of the current detector system 8

2.2 Rogowski coil 9

$\begin{array}{lll}2.3 & \text { Equivalent circuit diagram of the Rogowski coil }\end{array}$

2.4 Simplified equivalent circuit diagram of the Rogowski coil 11

2.5 Magnetic flux density, $B$ vs. current, $I \quad 12$

2.6 Magnetic flux density, $B$ vs. average core radius, $a \quad 13$

$\begin{array}{lll}2.7 & \text { No. of turns, } N \text { vs. average core radius, } a & 13\end{array}$

$\begin{array}{lll}2.8 & \text { Induced emf vs. no. of turns, } N & 14\end{array}$

2.9 Required number of turns, $N$ vs. current in conductor, $I \quad 15$

2.10 Rogowski coil used in the current detector 16

2.11 Equivalent circuit diagram of the Rogowski coil used in the current 16 detector

2.12 Impedance matching circuit used in the current detector 17

$\begin{array}{lll}2.13 & \text { V-I characteristic curve of a metal oxide varistor } & 18\end{array}$

$\begin{array}{lll}2.14 & \text { Symbol for metal oxide varistor } & 18\end{array}$

$\begin{array}{ll}2.15 & \text { A circuit with a full wave rectifier }\end{array}$

$\begin{array}{lll}2.16 & \text { Full wave rectification output } & 19\end{array}$

2.17 RC integrator involving a resistor and a capacitor 20

2.18 Circuit diagram of the current detector 21 
$\begin{array}{lll}3.1 & \text { Block diagram of the vibration detector } & 24\end{array}$

3.2 Accelerometer based on sensing the force on a mass 24

$\begin{array}{lll}3.3 & \text { Capacitive acceleration sensor }\end{array}$

$\begin{array}{lll}3.4 & \text { Accelerometer based on strain gauges } & 25\end{array}$

3.5 Magnetic accelerometer 25

$\begin{array}{lll}3.6 & \text { Heated gas accelerometer } & 26\end{array}$

3.7 MEMSIC2125 accelerometer 27

3.8 MEMSIC2125 pin diagram 28

$\begin{array}{lll}3.9 & \text { Inside view of the MEMSIC2125 }\end{array}$

3.10 Arduino UNO microcontroller board 30

3.11 Circuit diagram of the vibration detector 31

3.12 Flowchart for vibration detection 32

4.1 Block diagram of the lightning strike detector 33

4.2 Flowchart for the lightning strike detector 34

4.3 Sketch of the lightning strike detector output 35

$\begin{array}{lll}5.1 & \text { Frequency response of the current detector } & 37\end{array}$

$5.2 \quad$ Frequency response of the current detector (circuit simulation) 39

$\begin{array}{lll}5.3 & \text { Simulated sinusoidal input current } & 40\end{array}$

5.4 Induced emf across the Rogowski coil with sinusoidal input current 40

5.5 Output voltage of the current detector with sinusoidal current 41

5.6 Simulated damped sinusoidal current 41

5.7 Induced emf across the Rogowski coil with damped sinusoidal 42 current

5.8 Output voltage with the damped sinusoidal current 42

$5.9 \quad$ Simulated sinusoidal input current 43

5.10 Induced emf across the Rogowski coil with sinusoidal input current 43

5.11 Output voltage of the current detector with sinusoidal current 44

5.12 Simulated damped sinusoidal current 44

$\begin{array}{lll}5.13 & \text { Induced emf across the Rogowski coil }\end{array}$ 
5.14 Output voltage with the damped sinusoidal current 45

$\begin{array}{lll}5.15 & \text { Simulated transient current } & 46\end{array}$

5.16 Output voltage with the transient current 46

$\begin{array}{lll}5.17 & \text { Experimental setup } & 47\end{array}$

5.18 Plot of the induced emf (y-axis) against no. of turns of the coil (x- 48 axis)

5.19 Plot of the output voltage (y-axis) against current I (x-axis) 48

5.20 The constant sinusoidal input current used in the experimental 49 setup (left) and the simulation (right)

5.21 Induced emf across the Rogowski coil with sinusoidal input current 49 for the experimental setup (left) and the simulation (right)

5.22 Output of the current detector with sinusoidal input current for the 50 experimental setup (left) and the simulation (right)

5.23 Transient input current for the experimental setup (left) and the 51 simulation (right)

5.24 Output of the current detector with transient current for the 51 experimental setup (left) and the simulation (right)

5.25 Duty cyle (y-axis) against the acceleration (x-axis) 52

5.26 Serial monitor results for the vibration detector 53

5.27 Serial monitor results for the lightning strike detector only when 54 vibration is detected

5.28 Serial monitor results for the lightning strike detector only when current is detected

5.29 Serial monitor results for the lightning strike detector when both the current and vibration is detected 


\section{CHAPTER I}

\section{INTRODUCTION}

Lightning strikes can cause damage to transmission towers and lines and structural monitoring is a necessary step in determining the condition of the structures. There have been cases in which towers failed due to a broken member attributable to lightning damage [1]. Figure 1.1 shows a tower, the member of which broke due to lightning damage. The member did not break at the instant of the lightning damage but eventually failed due to fatigue and the absence of vibration dampers. Thus, a lightning strike detector is necessary for condition monitoring in transmission towers in order to ensure that such events do not take place and is essential in any transmission tower monitoring network.

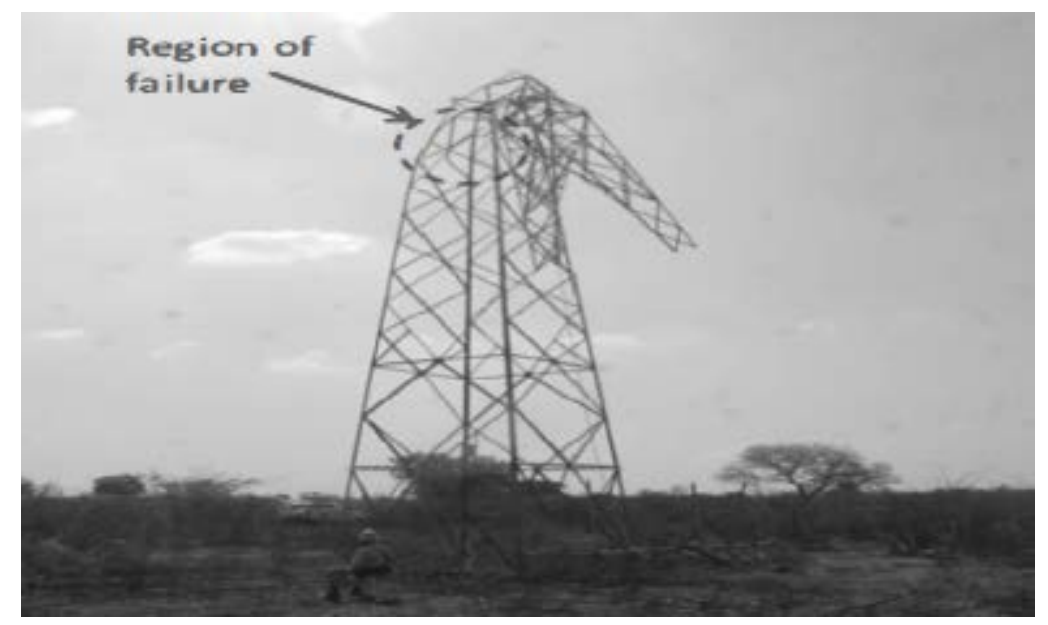

Figure 1.1: A tower that failed under broken member conditions [1]

\subsection{Overview}

In the past, many different methods have been proposed for the detection of lightning strikes. Some of these included the use of current transformers [2-4] for 
lightning current detection and measurement whereas others focused on electromagnetic radiation and sound waves in air, which can end up being quite expensive [5] and cannot distinguish strikes directly to towers or lines from strikes in the vicinity.

There are various researches which focused on the detection of acoustic signals in addition to other parameters. Yanjie et al. [5] proposed a method which made use of light and infrasound of lightning to accomplish the detection of lightning. Optical and acoustic sensors were used for this purpose. Ibrahim and Malek [6] used the time-tothunder method to determine the lightning strike distance via the implementation of a broadband antenna to detect the electric field wave and a microphone to detect the acoustic signature produced by the lightning strike. The electric field waves propagate at the speed of light whereas the acoustic waves propagate at the speed of sound. The arrival time-delay between the two signals was then used to calculate the distance to the lightning strike. Shanqiang et al. [7] discussed a thunder signal recognition method by analyzing acoustic signals obtained from multiple microphone arrays installed at the top platform on a seven-floor building. Although they were able to determine the thunder signal from the acoustic signals, some noise signals such as birds and whistles were mistaken as thunder signals by their proposed system.

Researches are also prevalent that make use of radio navigation system such as the GPS to aid in determining the location of lightning strikes. Cui et al. [8] focused on the development of a lightning radiation electromagnetic field data acquisition system based on a GPS receiver, counter, data acquisition card and lightning detection stations. Ibrahim and Ghazali [9] presented a lightning detection system using a combination of magnetics direction finder and time of arrival sensor which provided both real-time and historical lightning data. 
Other researches are based on the detection of current that results from a lightning strike. There are many different methods for the detection and/or measurement of current. A small resistor can be connected in series with a circuit in which the current should be monitored. The voltage obtained across the resistor would be proportional to the current. Another method, used in the case of AC current, is by using a coil in a current transformer arrangement, as shown in Figure 1.2. A coil with $\mathrm{N}$ turns is placed around the current carrying conductor. The coil acts as the secondary coil of the current transformer with the current carrying conductor acting as the (single-turn) primary coil. An air-cored coil used in current measurement applications is called a Rogowski coil.

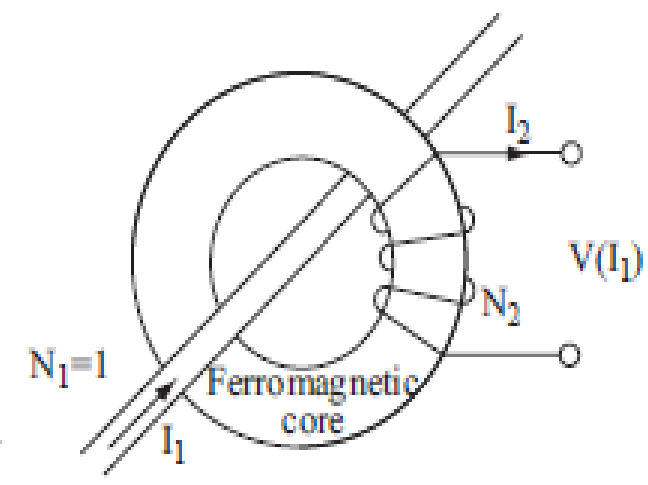

Figure 1.2: A coil acting as the secondary coil of a current transformer [10]

The Rogowski coil has been used for the detection of lightning current in various researches. Kawabata et al. [2] presented the usage of Rogowski coils to measure lightning current waveforms in tall structures, such as wind turbines and antenna towers. Their work focused on the development of a shunt lightning current measuring system using a Rogowski coil which can be used to measure the current which flows close to the electrical equipment, in large structures like wind turbines, and could result in their damage. Han et al. [3] designed a printed circuit board Rogowski coil that could be used for measuring nanosecond risetime pulsed currents. Li et al. [4] used a 
Rogowski coil with an external integrator for measurement and location of lightning current, up to $400 \mathrm{kA}$ on the transmission line. Although the current detection method can be quite reliable for accurate detection of lightning strikes on structures, a double detection method is often desired, in order to avoid false positives. A change in polarity of the input current might also lead to incorrect results in the case of detection systems that detect current using Rogowski coils [11]. Hence, the current detection method should be reinforced with a secondary detection technique such as MEMS (Microelectromechanical Systems) sensors for vibration detection [12, 13].

MEMS sensors are used for structural vibration and seismic motion detection purposes. Kon and Horowitz [12] fabricated a ZnO piezoelectric microsensor on silicon and steel substrates. The sensors were used for structural vibration monitoring and as they were developed using microelectromechanical systems (MEMS) technology, they did not put an extra burden on the structures on which they were placed. Deng et al. [13] used an electrochemical vibration sensor for seismic motion monitoring. The proposed vibration sensor could function in places where lowfrequency seismic motion detection was required. Sabato [14] used a wireless MEMS accelerometer board for pedestrian bridge vibration monitoring.

\subsection{Objectives of the thesis}

This thesis discusses a lightning strike detector with current and vibration detection capabilities. Each tower in a network can be equipped with the lightning strike detection system which can then be used to determine whether or not any lightning strike occurred in a transmission line or tower.

Figure 1.3 and 1.4 depicts possible scenarios regarding the propagation of current and shockwaves during the moment of the strike [15]. Figure 1.3 shows the current propagation path after a lightning strike in a transmission tower. Although the 
lightning struck the transmission tower $\mathrm{A}$, the current is flowing to ground through both $\mathrm{A}$ and $\mathrm{B}$.

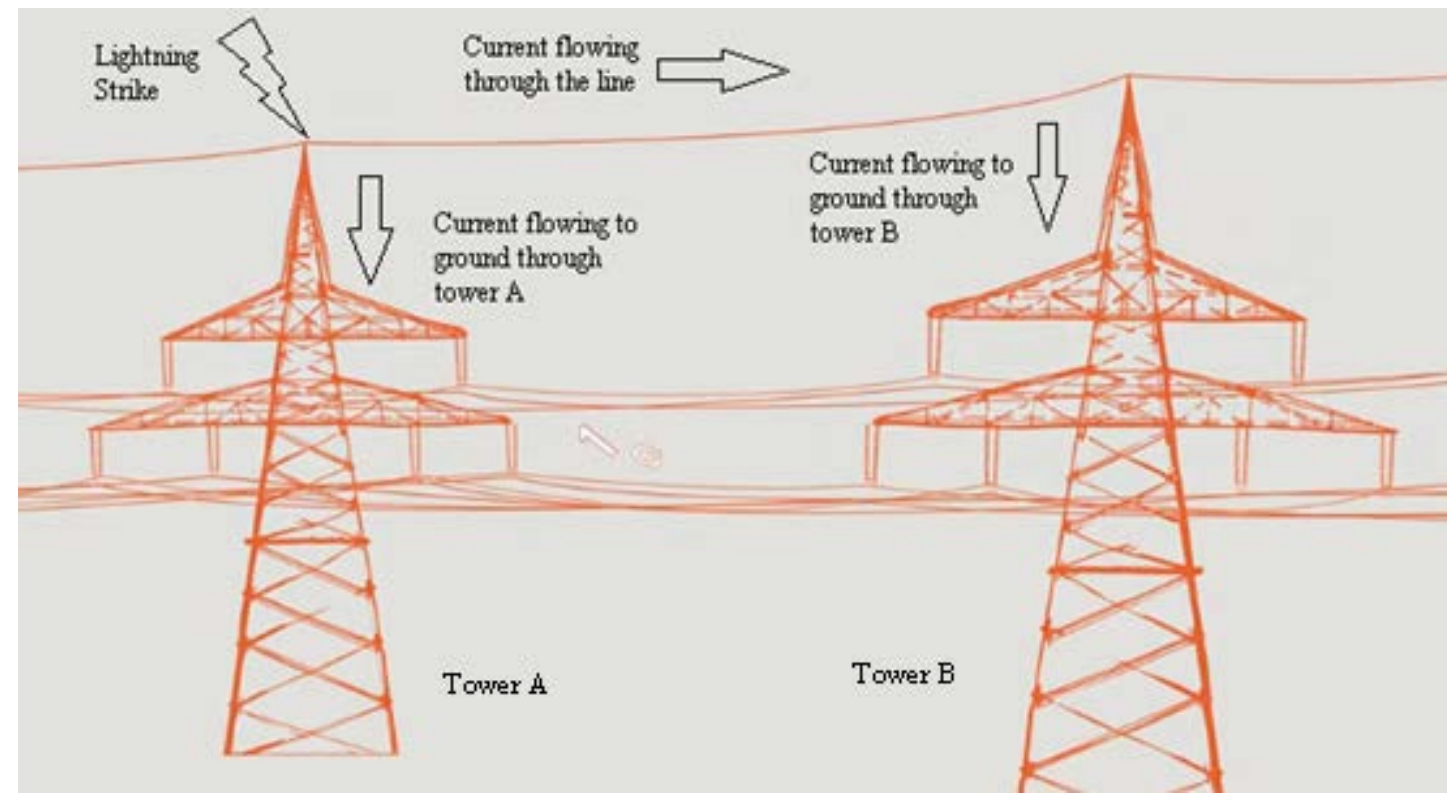

Figure 1.3: Current propagation during a lightning strike [15]

Figure 1.4 shows the shockwave propagation through various towers during the instant of the lightning strike. As the lightning is striking the Tower A directly, it will register a high shockwave whereas in Tower B, only a vibration effect will be felt. A current due to a lightning strike may not flow in this case. Thus, the lightning strike detector is programmed to make use of both the current and vibration notification to effectively pinpoint the structure of interest. 


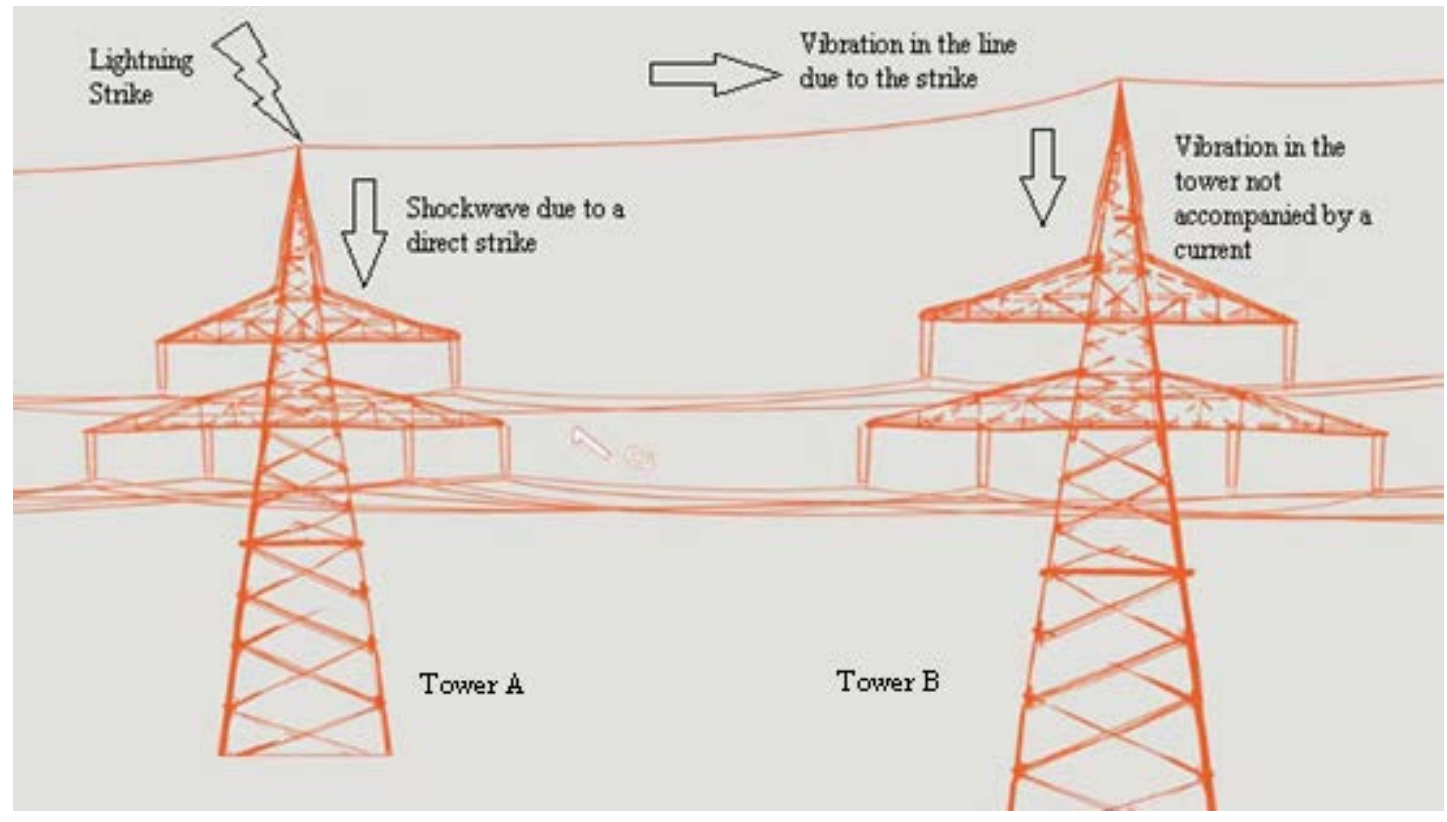

Figure 1.4: Shockwave propagation during a lightning strike [15]

The aim of the thesis is to make use of the lightning current as well as the vibration caused by the lightning strike. This double detection measure ensures the proper detection of a lightning strike, reduces the chances of false detection and allows for determination of the nearest tower. The lightning strike detector is comprised of a current detector and vibration detector. The current detector was built using a Rogowski coil, as mentioned in the overview section, which was reinforced with additional components for proper detection of a lightning current. The vibration detector was built using an accelerometer.

The Rogowski coil is an air cored coil that can be placed around a member of the tower in a toroidal fashion to detect current to ground due to lightning. The magnetic field produced by current in the member during a lightning strike induces a voltage in the coil. If the Rogowski coil is placed around the ground wire for the tower, it can also be used to detect fault currents to ground. A voltage develops across the coil, proportional to the current [10]. The detection of current confirms the presence of a strike but it does not give an indication regarding which tower is closest since currents 
are expected to flow to ground through multiple towers during a lightning strike. To resolve this, an accelerometer is attached to each tower. The vibration of the tower at the moment of the strike is useful in determining the time correlation between the strike and the current detection. In order to differentiate between vibration due to lightning strikes and that due to other causes such as high wind speeds, the detection is only flagged for vibrations that are preceded by a current being detected by the Rogowski coil.

\subsection{Thesis organization}

After a basic introduction and overview in Chapter 1, the current detector system and its various components are described in Chapter 2. Similarly, in Chapter 3, the vibration detector system is described. The combined lightning strike detector is shown in Chapter 4. The results are given in Chapter 5. A summary of the thesis and ideas for extension of the thesis work are given in the last chapter. 


\section{CHAPTER II}

\section{CURRENT DETECTOR}

This chapter discusses the current detector system developed to detect the lightning current that emanates from a lightning strike, in a member of a transmission tower. The current detector consists of a Rogowski coil, used as a current transformer, and associated circuitry. The coil can be opened and placed over a conductor through which the current is expected to flow. The coil can be placed around any member of a transmission tower or the grounding wire to determine the occurrence of lightning strikes. All the towers in a network of towers would be equipped with this device.

\subsection{Block diagram of the proposed current detector}

Figures 2.1 shows the block diagram of the current detector. The input current is detected by the Rogowski coil and an RC circuit is used for impedance matching. A metal oxide varistor or zener diodes (MOV) is used for safety measures and a full wave rectifier is used to ensure that the output always stays positive. An RC integrator is then placed after which the output is obtained. A large resistor is also placed across the output to ensure that the capacitor is properly discharged after each lightning strike event. A zener diode is placed in the output as a safety measure. The blocks of the block diagram are explained in sections 2.2-2.5.

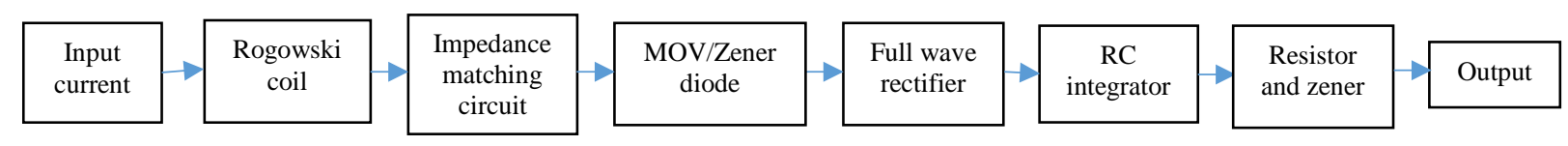

Figure 2.1: Block diagram of the current detector system 


\subsection{Rogowski coil}

The Rogowski coil is an air-cored flexible coil capable of measuring ac and impulse currents. It was named after German physicist Walter Rogowski. The use of the Rogowski coil has a number of advantages: 1) No saturation at possibly large currents, 2) Not affected by dc, 3) Electrically safe when open, 4) Smaller size and lower weight, 5) Simplicity and flexibility, 6) Low cost, 7) Linear device, 8) No issue with magnetization and magnetization errors [19].

Equations governing the behavior of the Rogowski coil can be derived which can then be used to figure out its characteristics properly before designing a coil specifically for an application. Figure 2.2 shows a Rogowski coil with an average “core” radius $a$, radius of turns $b, N$ turns, electrical resistivity of the coil wire $\rho_{\mathrm{c}}$, length of coil wire $l_{w}$, the radius of the wire $d$ and vacuum permittivity of $\varepsilon_{0}$. The coil is placed around a current carrying wire producing a magnetic flux density $B$ [10].

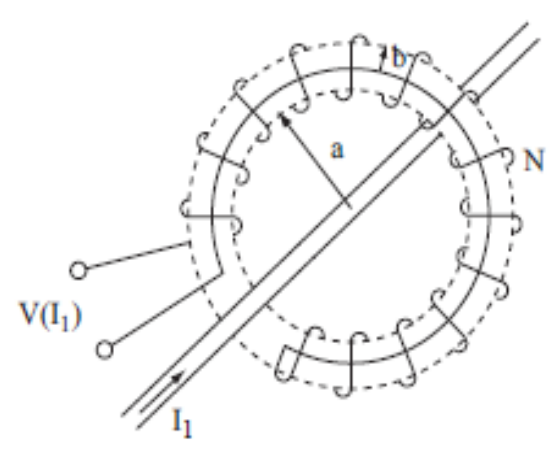

Figure 2.2: Rogowski coil [10]

The Rogowski coil does not have a core and therefore the permeability is $\mu_{0}$ (the permittivity of air or the surrounding plastic material). The magnetic flux density, $B$, at the center of the coil is given by (1). In order to derive the formula for the emf in the coil, the flux needs to be calculated. The flux, $\phi$, in the cross sectional area of the coil 
is given by (2). Given a time dependent current $I$ at angular frequency $\omega$, the emf induced in the coil is given by (3) and (4) where $M$ is the mutual inductance of the coil.

$$
\begin{gathered}
B=\frac{\mu_{0} I}{2 \pi a} \\
\phi=\int_{s} B \cdot d s \approx B S=B \pi b^{2}=\frac{\mu_{0} I b^{2}}{2 a} \\
e m f=N \frac{d \phi}{d t}=\left(N \frac{\mu_{0} b^{2} \omega}{2 a}\right) I_{0} \cos (\omega t) \\
e m f=M \frac{d I}{d t}
\end{gathered}
$$

Figure 2.3 shows the equivalent circuit diagram of the Rogowski coil where Vrc is the input voltage to the Rogowski coil, $L_{S}$ is the self-inductance of the coil, $R_{S}$ is equivalent resistance of the coil, $C_{S}$ is stray capacitance and $C_{P}$ is turn to turn capacitance [4]. $Z$ is the output impedance of the Rogowski coil and Vout is the output voltage. $C_{P}$ can be ignored compared with $C_{S}$ and thus the circuit can be reduced to that in Figure 2.4 [4]. The parameters of the equivalent circuit are given by (5)-(8) $[16,17]$. These were used initially to determine the behavior and efficacy of the Rogowski coil via simulation in LTspice before practical implementation. The transfer function of the simplified equivalent circuit is given by (9).

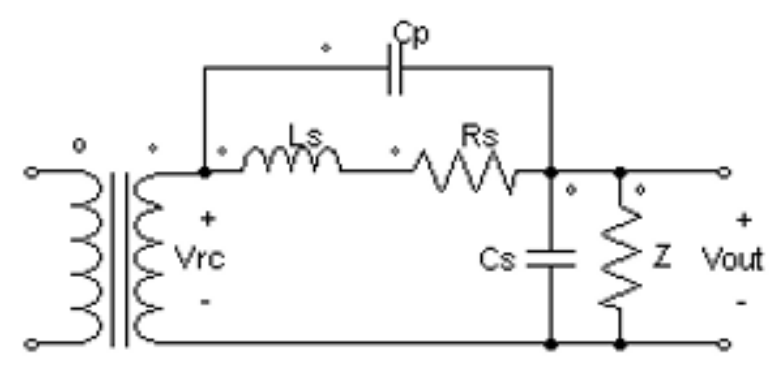

Figure 2.3: Equivalent circuit diagram of the Rogowski coil 


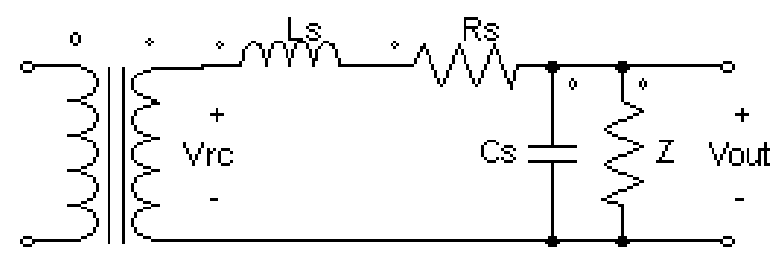

Figure 2.4: Simplified equivalent circuit diagram of the Rogowski coil

$$
\begin{gathered}
R_{S}=\rho_{C} \frac{l_{w}}{\pi d^{2}} \\
L_{S}=\frac{\mu_{0} N^{2} b}{\pi} \log \frac{2 a+4 b}{2 a} \\
C_{S}=\frac{4 \pi^{2} \varepsilon_{0}(4 a+4 b)}{\log \left(\frac{4 a+4 b}{4 b}\right)} \\
N=\frac{\pi M}{\mu_{0} b \log \frac{2 a+4 b}{2 a}} \\
\frac{V_{\text {out }}}{V_{\text {rc }}}=\frac{Z}{s^{2} L_{S} Z C_{S}+s\left(L_{S}+R_{S} Z C_{S}\right)+\left(R_{S}+Z\right)}
\end{gathered}
$$

A number of simulations were done to understand how coil parameters affect the Rogowski coil. For these simulations, documented in Figures 2.5 through 2.9, the average core radius $\mathrm{a}=0.10 \mathrm{~m}$ and the radius of turns $\mathrm{b}=0.005 \mathrm{~m}$ are kept constant unless otherwise specified.

Figure 2.5 shows how the magnetic flux density varies around a current carrying conductor for the coil. The magnetic flux density increases with the magnitude of the current in the conductor in a linear fashion. A high current yields a high magnetic flux 
density. This shows why the induced emf is high in presence of a large current as it is proportional to the magnetic flux density.

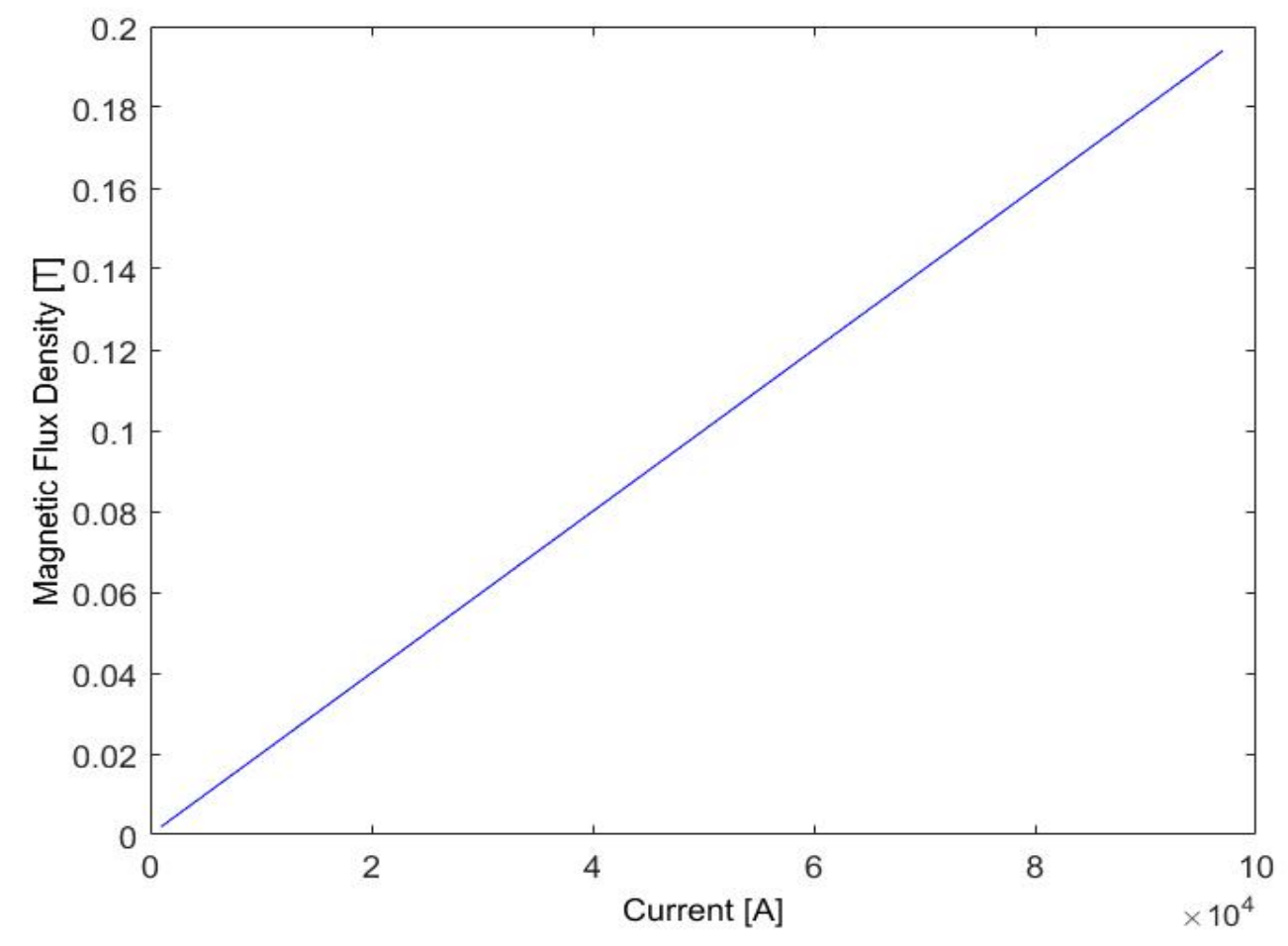

Figure 2.5: Magnetic flux density, $B$ vs. current, $I$

Figure 2.6 shows how magnetic flux density, $B$ varies with the average core radius, $a$ around a current carrying conductor. The average core radius $a$ was varied from $0.015 \mathrm{~m}$ to $0.15 \mathrm{~m}$. The magnetic flux density decreases with increasing the radius and thus, the radius should be kept as small as possible when designing the Rogowski coil as a smaller radius will yield a higher induced emf. 


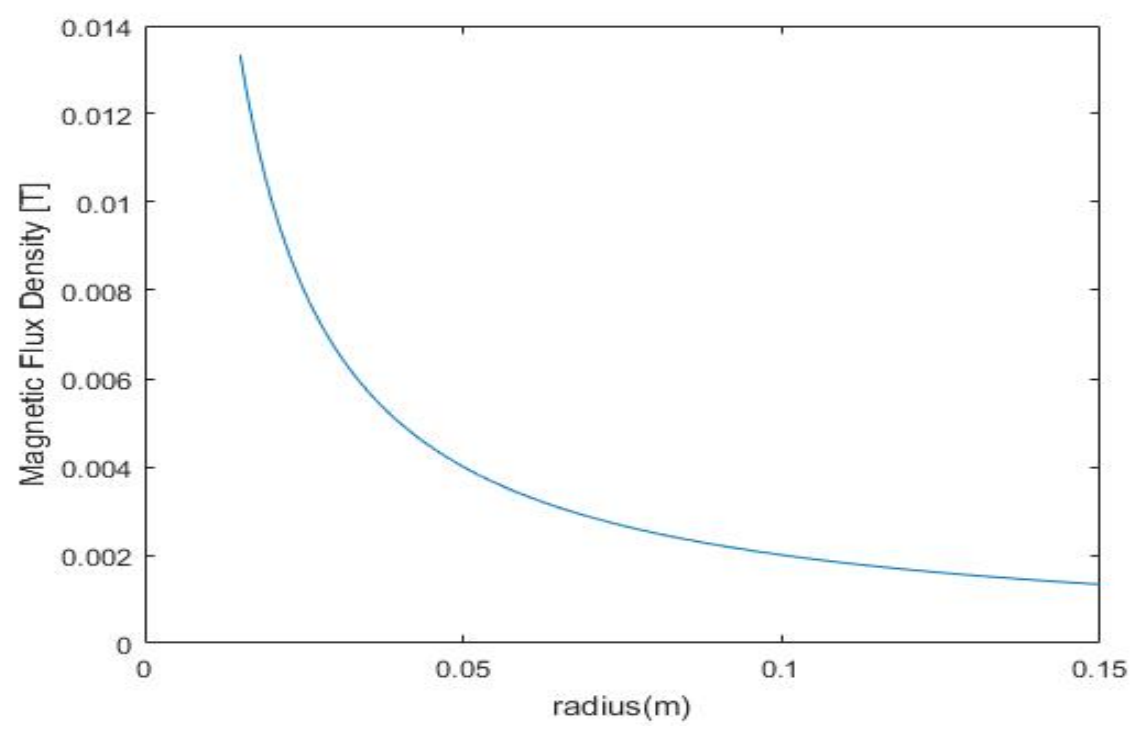

Figure 2.6: Magnetic flux density, $B$ vs. average core radius, $a$

Figure 2.7 shows that for a fixed induced emf of $0.5 \mathrm{~V}$, a linear relationship between the average core radius, $a$ and the number of turns, $N$ must be maintained. The average core radius $a$ was varied from $0.015 \mathrm{~m}$ to $0.15 \mathrm{~m}$. If the radius of the coil is increased, the number of turns of the coil would also need to be increased in order to have the same induced emf. This shows that when designing a coil, it is better to minimize its radius as it leads to a lower number of turns and thereby, less loss.

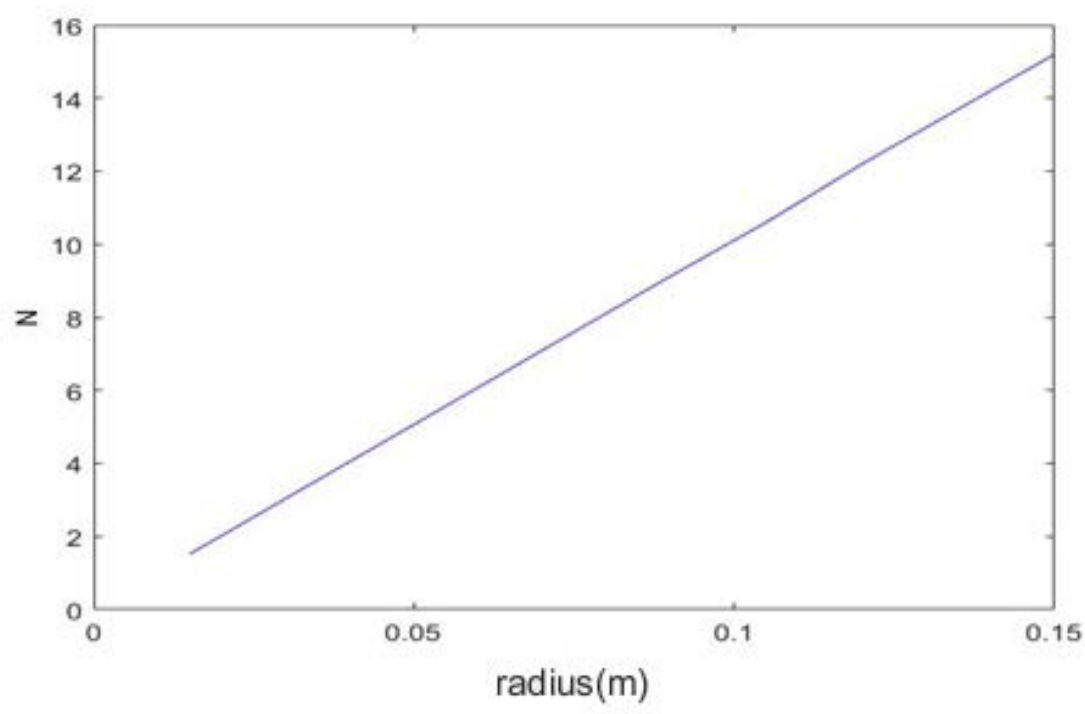

Figure 2.7: Number of turns, $\mathrm{N}$ vs. average core radius, $a$ 
Figure 2.8 shows the linear graph of induced emf vs. the number of turns of the Rogowski coil. For a particular current $I$, through the conductor passing through the coil, the number of turns can be increased in order to generate higher induced emf. As a small current was used in the prototype, the number of turns were increased in order to have sufficient emf.

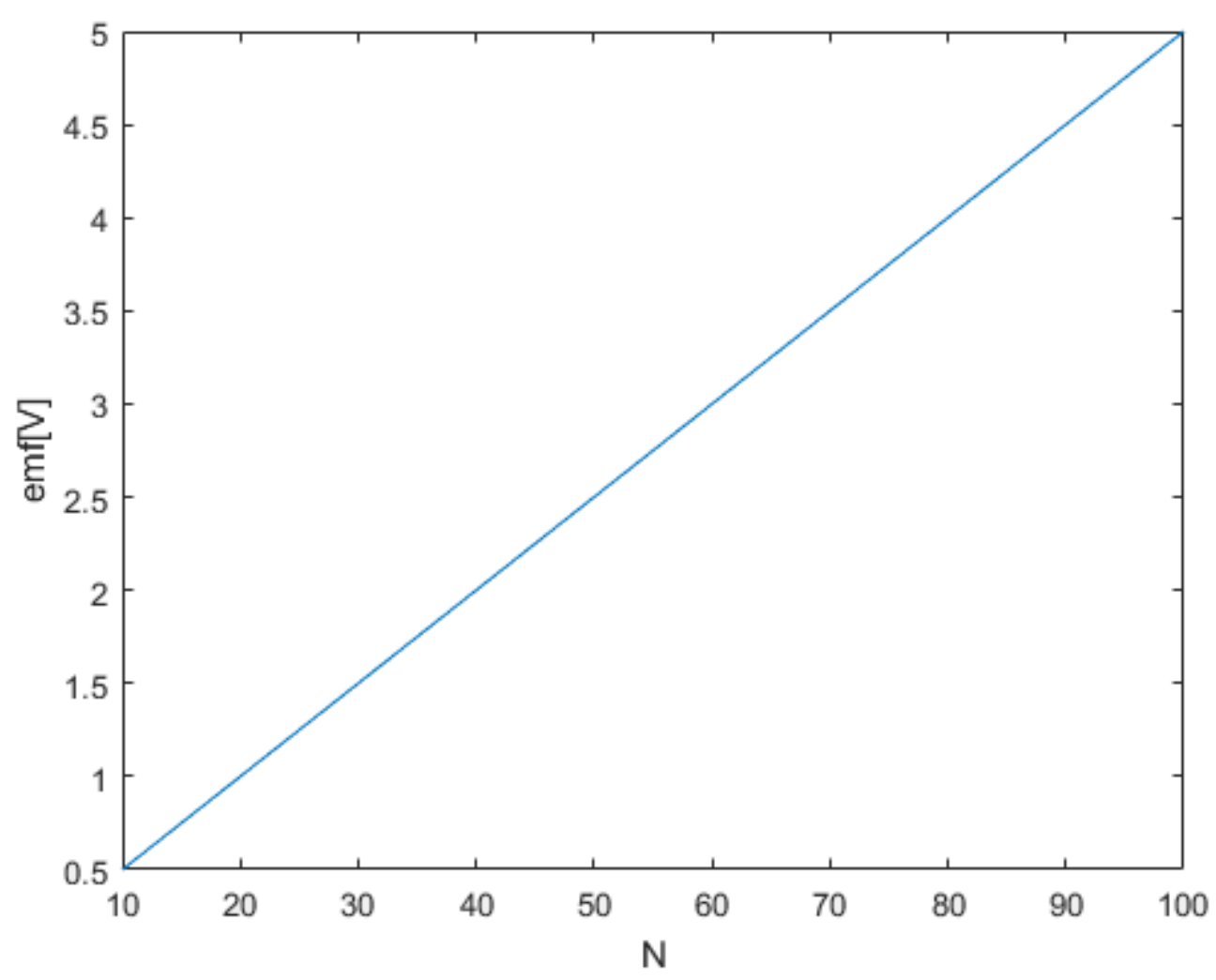

Figure 2.8: Induced emf vs. number of turns, $N$

Figure 2.9 shows the required number of turns in the Rogowski coil vs. current in conductor. The required number of turns decreases with increasing the magnitude of current. In case of a high current, the number of turns can be lowered to produce the desirable emf, assuming all the other parameters are constant. 


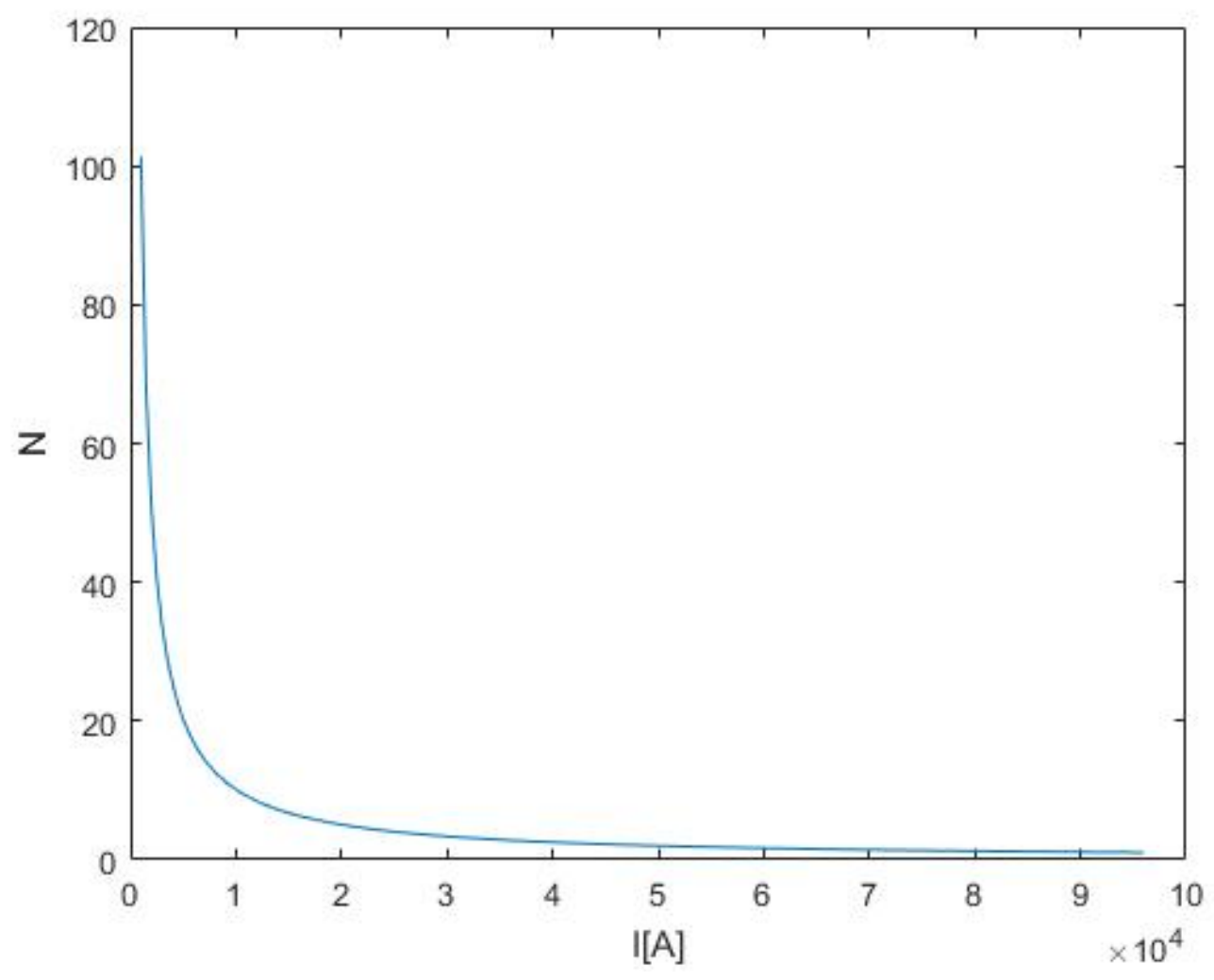

Figure 2.9: Required number of turns, $N$ vs. current in conductor, $I$

Figure 2.10 shows the Rogowski coil used for practical implementation which is just a circular plastic ring wound with copper wires. It shows a large number of turns, 1050, because it was used in a scaled laboratory prototype where the current was low. A large number of turns were used to generate sufficient emf. However, as the current in the actual field would be quite high, in the range of KA's, the number of turns can be significantly lowered to around 10-20 for a coil appropriate for use in lightning strike detection on an actual transmission tower. 


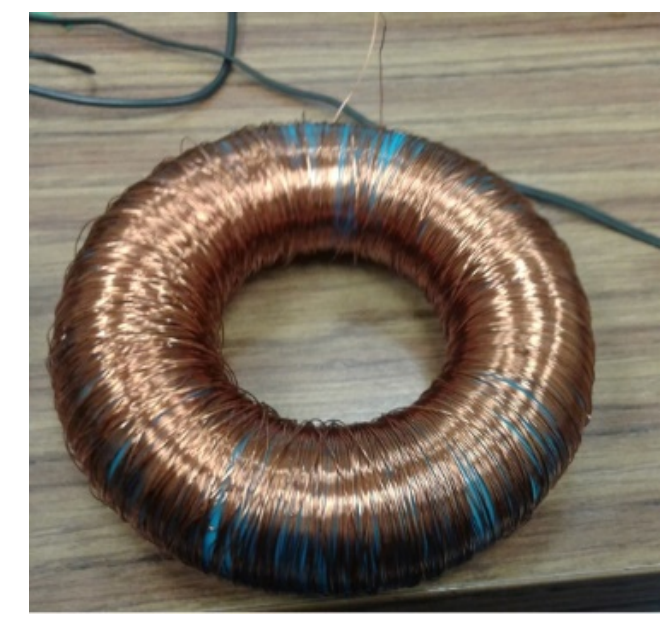

Figure 2.10: Rogowski coil used in the current detector

Figure 2.11 shows equivalent circuit of the Rogowski coil used in the current detector. The resistance, $R_{s}$ and inductance, $L_{s}$ of the Rogowski coil were found to be equal to $24.52 \Omega$ and $4.506 \mathrm{mH}$ respectively, using an RLC meter. In the measurement of the shunt impedance, only resistance values were obtained. The RLC meter did not show any capacitance value and thus, the capacitance was negligible thereby making the equivalent circuit of the Rogowski coil in Figure 2.11 different than the simplified equivalent circuit of the Rogowski coil, shown in Figure 2.4. The complex conjugate of the impedance of the Rogowski coil would need to be placed across the coil for impedance matching. The type of impedance used and the values required are given in section 2.6.

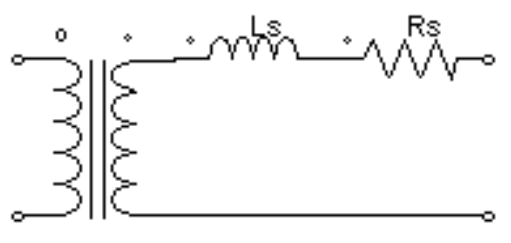

Figure 2.11: Equivalent circuit diagram of the Rogowski coil used in the current detector 
2.3 Impedance matching circuit

All devices have an internal impedance that needs to be matched for proper operation of the device. The internal impedance can be either real or complex. In case of many circuits, impedance matching via conjugate matching is required for either the transfer of maximum power from the source to the output or for ensuring nonreflection. For example, an amplifier will be required to have an impedance of $R$ $j X$ to transfer maximum power to a speaker of impedance $R+j X$. Impedance matching is also required for sensors and actuator operating at high frequencies. In the case of Rogowski coils, impedance matching is required to prevent reflection of voltage or current.

Figure 2.12 shows the impedance matching circuit for the current detector. For impedance matching, the complex conjugate of the impedance of the Rogowski coil consisting of resistance, $R_{m}$ of $24.52 \Omega$ and capacitance, $C_{m}$ of $5.618 \mathrm{uF}$ was placed across the Rogowski coil.

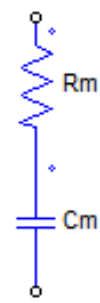

Figure 2.12: Impedance matching circuit used in the current detector

\subsection{Metal oxide varistor (MOV)}

A varistor (variable resistor) is a voltage-dependent resistor with a symmetrical V-I characteristic curve, as shown in Figure 2.13 [18]. In other words, it exhibits diode-like characteristics in both directions of a passing current and thus can be represented as two zener diodes facing towards each other, for the sake of simplicity and for SPICE 
simulation, as shown in Figure 2.14. Any voltage above 5 V was clipped. Due to the unavailability of MOV's rated at $5 \mathrm{~V}$, zener diodes were used in the prototype. The resistance of a varistor decreases with an increase in applied voltage. It is placed in parallel with the component that is to be protected. It forms a low-resistance shunt in case of an increased voltage, thereby preventing further rise in surge overvoltage.

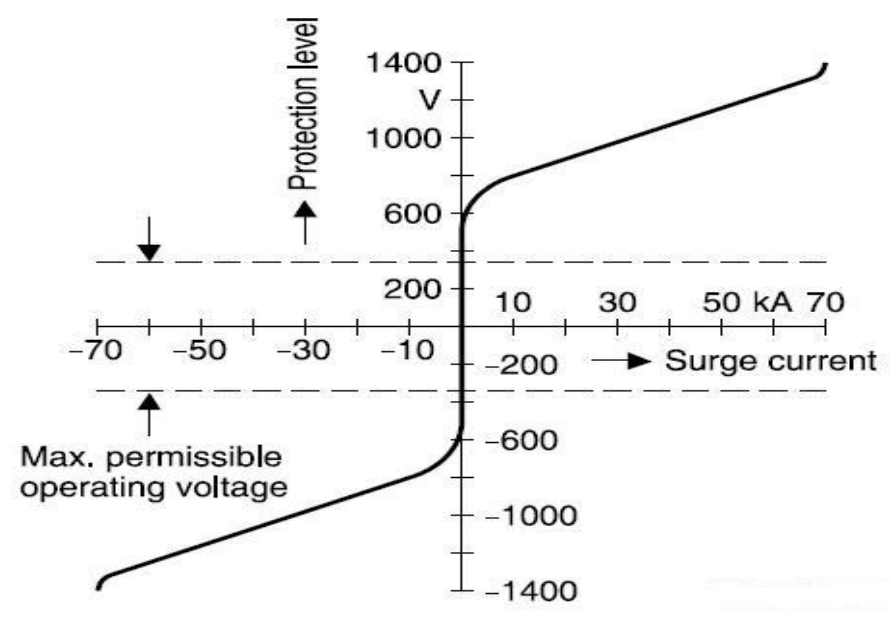

Figure 2.13: V-I characteristic curve of a metal oxide varistor [18]

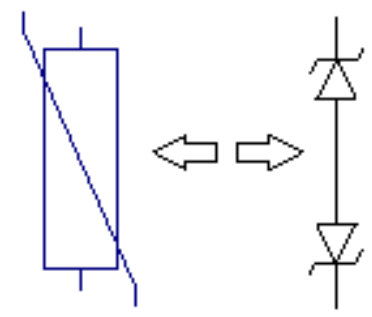

Figure 2.14: Symbol for metal oxide varistor

The voltage dependence of varistors is characterized by (11)

$$
I=K V^{\alpha}
$$

where $I$ denotes the current through the varistor, $K$ is a constant dependent on the type of varistor used, $V$ is the voltage across the varistor and $\alpha$ is the nonlinearity exponent. 


\subsection{Full wave rectifier}

The full wave rectifier, in the current detector, ensures that a reversal in the polarity of the current does not affect the operation of the circuit. This is important because the current detector's output is connected to a microprocessor as part of an integrated transmission line sensing system [11]. A circuit with a full wave rectifier, made up using diodes $\mathrm{D}_{1}, \mathrm{D}_{2}, \mathrm{D}_{3}$ and $\mathrm{D}_{4}$, is shown in Figure 2.15. During the positive halfcycle of the input voltage, the power is supplied to the load through diodes $D_{1}$ and $D_{2}$. During the negative cycle, diodes $\mathrm{D}_{3}$ and $\mathrm{D}_{4}$ conduct. Thus, an output is produced which can be positive and zero, but never negative as shown in Figure 2.16 [19]. The capacitor at the output acts as a smoothing capacitor which regulates the rippled output of the rectifier into a smooth DC voltage.

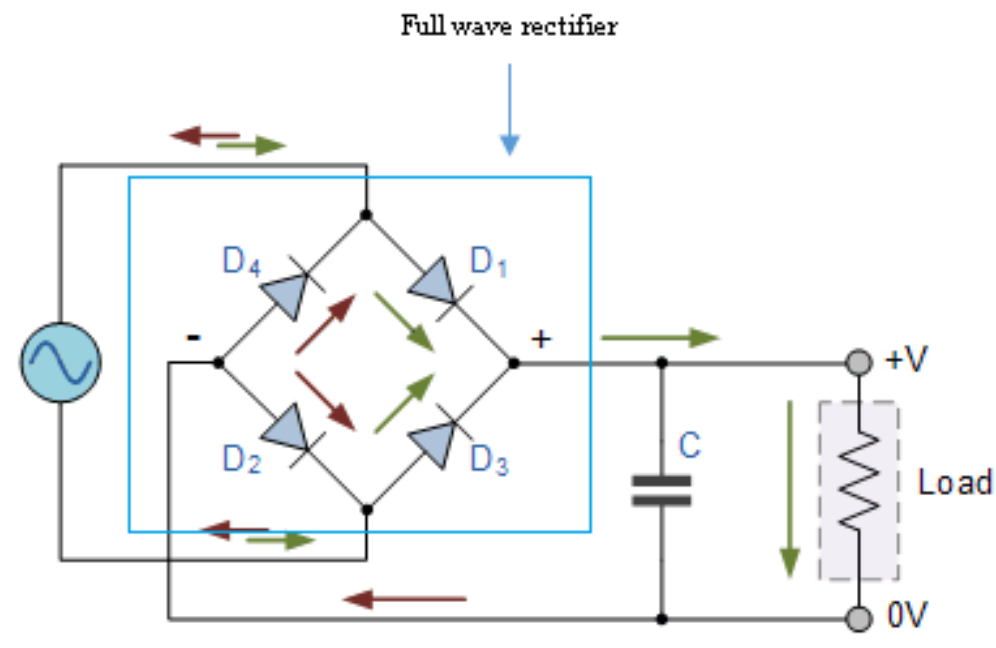

Figure 2.15: A circuit with a full wave rectifier [19]

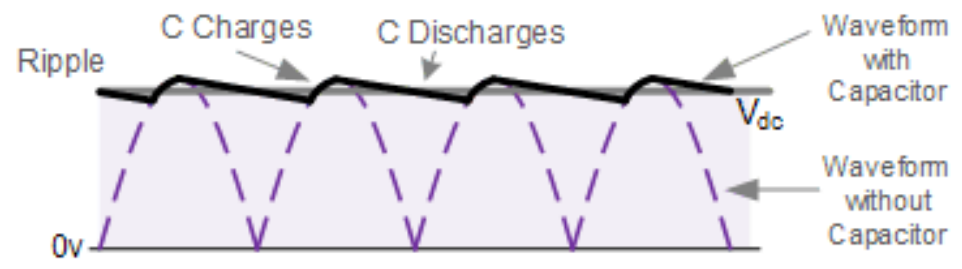

Figure 2.16: Full wave rectification output [19] 


\subsection{RC integrator}

An integrator (active using an op-amp, or passive) can be used to provide a voltage which recreates the current waveform. The integrator needs to be designed properly in order to ensure that saturation does not take place and the output voltage remains linear with the input current. For frequencies approaching a few hundred kilohertz, bandwidth limitations of the op-amp used for integration can become significant making active integration difficult. Hence, a passive RC integrator was used for the current detector [20]. Figure 2.17 shows an RC integrator that was used as a low pass filter in the current detector to eliminate transients. The emf is integrated using an RC integrator to eliminate transients. A small integrating resistor was used for this purpose (only the resistance of the connecting wire). Low resistance of integrating resistor can increase upper cut-off frequency and decrease lower cut-off frequency [21]. The capacitor of the RC integrator also acts as a smoothing capacitor for the full wave rectifier and helps in regulating the output voltage to a constant dc value.

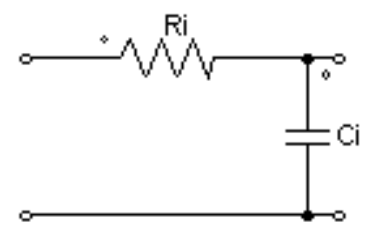

Figure 2.17: RC integrator involving a resistor and a capacitor

\subsection{Proposed current detector}

Figure 2.18 shows the circuit diagram of the system. The circuit was built on a breadboard. The resistance, $R_{s}$ and inductance, $L_{s}$ of the Rogowski coil were equal to $24.52 \Omega$ and $4.506 \mathrm{mH}$ respectively. For impedance matching, the complex conjugate of the impedance of the Rogowski coil, made of resistance, $R_{m}$ of $24.52 \Omega$ and capacitance, $C_{m}$ of $5.618 \mathrm{uF}$ was placed across the coil. $R_{i}$ of $100 \mathrm{~m} \Omega$ and $C_{i}$ of $10 \mathrm{nF}$ are the integral parameters. The integral parameters were chosen on the basis of trial 
and error to obtain a smooth waveform. A large $R_{d}$ value of $1 \mathrm{M} \Omega$ is at the output used to ensure the proper discharge of the capacitor once it is fully charged. A zener diode is also used for safety as a sort of double protection measure. This will provide protection to the microcontroller incase the MOV/Zener diodes stop working. $M$ is the mutual inductance of the coil. The transfer function, $H(s)=V(s) / I(s)$ of the current detector is given by (11) where $V(s)$ is the output voltage and $I(s)$ is the input current, and was used to plot the frequency response of the system, given in Chapter 5.

$$
H(s)=\frac{M R_{d}\left(C_{m} R_{m} s^{2}+s\right)}{L_{s} C_{m} C_{i} R_{m} R_{d} s^{3}+\left(L_{s} C_{m} R_{m}+L_{s} C_{m} R_{d}\right) s^{2}+\left(C_{m} R_{m} R_{s}+L_{s}\right) s+R_{s}}
$$

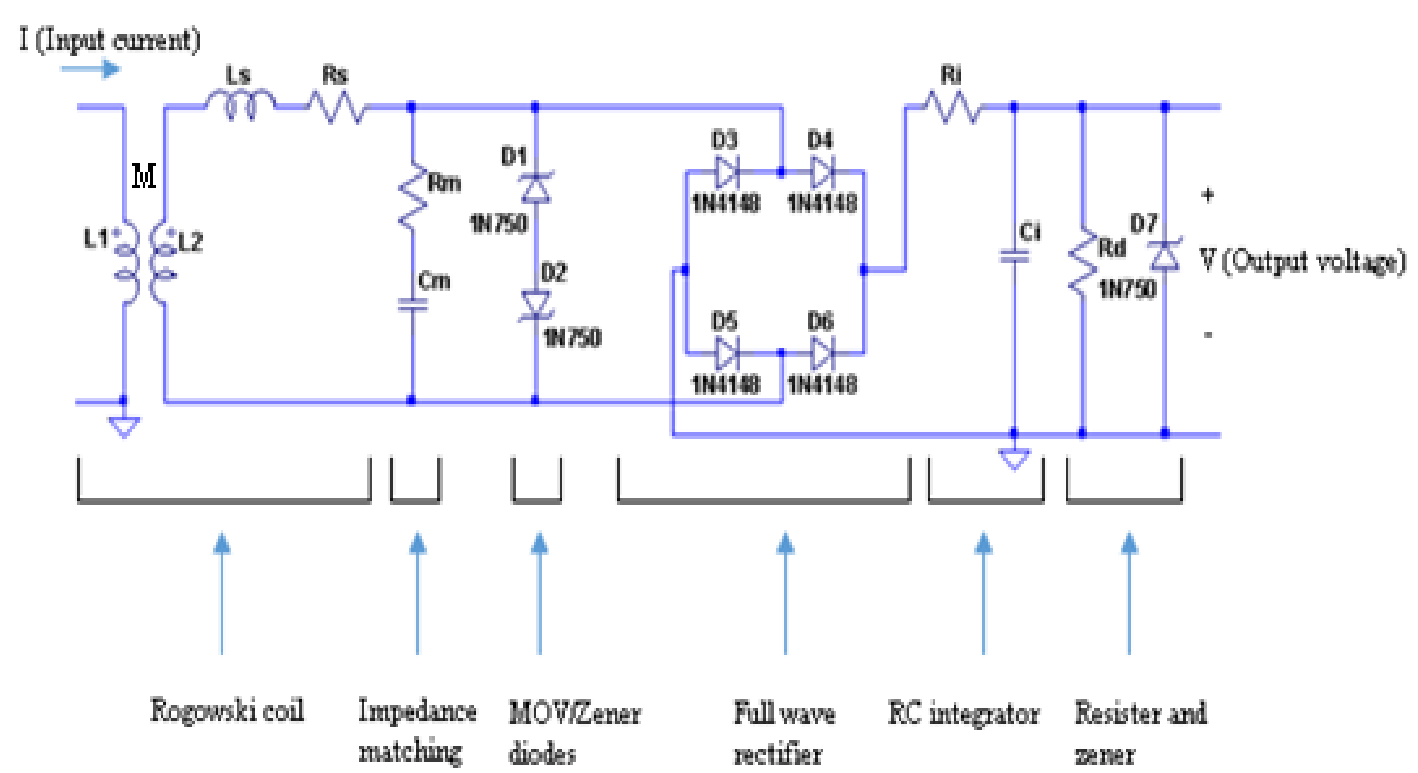

Figure 2.18: Circuit diagram of the current detector

\subsection{Summary}

The current detector and its various components are discussed in this chapter. A Rogowski coil is placed across a conductor through which the current is flowing. An MOV was used for voltage protection. A full wave rectifier was used to ensure that 
the polarity of the input current does not affect the behavior of the coil and RC integrator was used to eliminate transients. A large resistor was placed in the output to ensure that the output capacitor was fully discharged and an additional zener diode was placed for further protection. The current detector will be unified with the vibration detector, discussed in the next chapter, to make up the combined lightning strike detector. 


\section{CHAPTER III}

\section{VIBRATION DETECTOR}

This chapter discusses the vibration detector, and its various components, which is required to detect the vibration at the instant of the lightning strike. The vibration detector prototype was built using MEMSIC2125, a 2-axis accelerometer, connected to an Arduino microcontroller board.

\subsection{Block diagram of the proposed vibration detector}

Figure 3.1 shows the block diagram of the vibration detector. Although there are different ways of interfacing microcontrollers, the particular device selected features PWM outputs. This type of output is easily interfaced to a microcontroller. The dualaxis accelerometer has two output pins (one for the x-axis and the other for the y-axis). Any movement or acceleration experienced in both the x-axis and the y-axis is detected by the accelerometer. The duty cycle of output pulses is a measure of the acceleration experienced on a particular axis with 50\% duty cycle at zero acceleration. The duty cycle of the output pulses was read using the microcontroller to determine the acceleration. The microcontroller was used to calibrate the maximum and minimum values of the acceleration experienced in both the x-axis and the $y$-axis. The microcontroller was then connected to a computer using a USB jack to obtain the output. If an acceleration detected in either the x-axis and/or the $y$-axis exceeds the maximum acceleration or goes below the minimum acceleration, a vibration notification is given by the vibration detector. The low range is an advantage in the present application since the vibrations caused by the lightning strike is expected to be 
low, except for direct strikes to the tower. If there is a lightning impact on the line, then there is a possibility that the nearest tower will only receive a minor vibration. Thus, an accelerometer with a rather limited range was deemed appropriate.

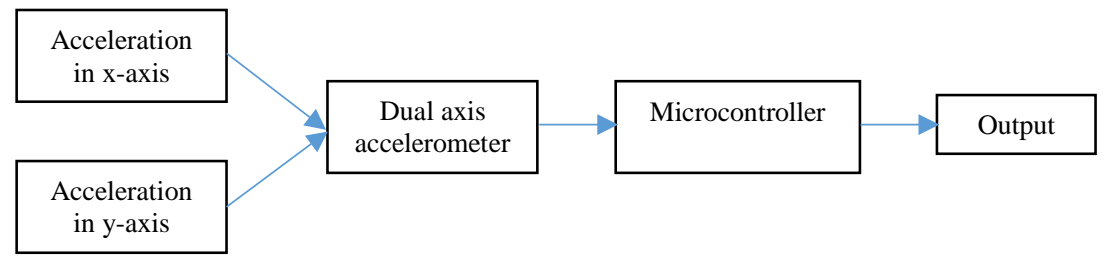

Figure 3.1: Block diagram of the vibration detector

\subsection{Accelerometer}

Acceleration can be detected by measuring the force on a mass, courtesy of Newton's second law of motion $(\mathrm{F}=\mathrm{ma})$. In the rest position, the acceleration is zero due to the absence of a force being exerted on the mass. For a mass $\mathrm{M}$, the force on the mass is directly proportional to acceleration. Figure 3.2 shows an accelerometer based on this principle [10].

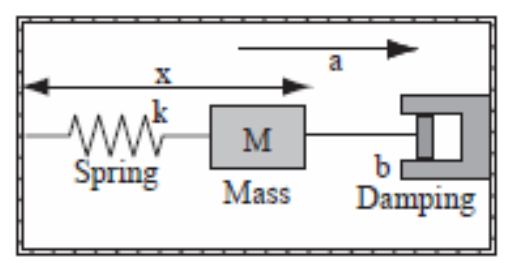

Figure 3.2: Accelerometer based on sensing the force on a mass [10]

There are also capacitive accelerometers where one plate of a small capacitor is fixed and connected to the base of the sensing device. The second plate is allowed to move freely. The acceleration on the sensor results in a change in capacitance. Figure 3.3 shows one such accelerometer [10]. 


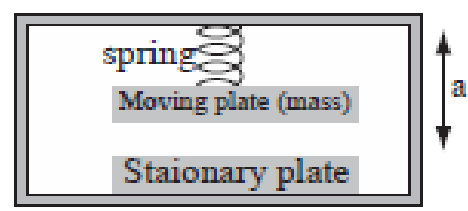

Figure 3.3: Capacitive acceleration sensor [10]

Accelerometers are also available which use strain gauges to measure the strain due to acceleration as shown in Figure 3.4. A mass is suspended on a cantilever beam and a strain gauge senses the bending of the beam. A second strain gauge may be fitted under the beam to make it a dual axis accelerometer.

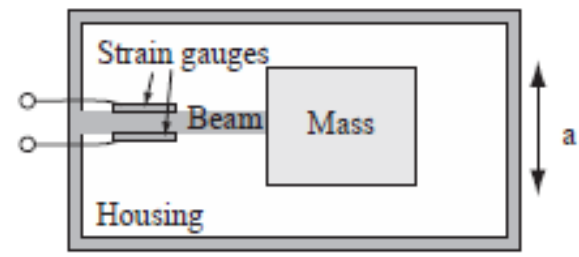

Figure 3.4: Accelerometer based on strain gauges [10]

A magnetic accelerometer can be built as a variable inductance device in which the mass or part of the mass is placed inside a coil as shown in Figure 3.5. The inductance of the coil is proportional to the position of the mass and the further the ferromagnetic rod is inside the coil, the higher is the inductance [10].

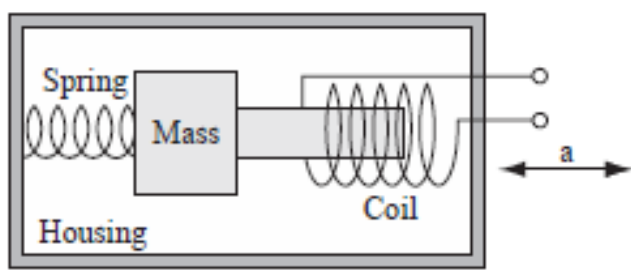

Figure 3.5: Magnetic accelerometer [10]

A heated gas accelerometer, as shown in Figure 3.6, works on the basis of heat transfer by natural convection. A single heat source, a chamber of gas, is placed in the 
middle of the accelerometer and thermopiles are placed on two (or more) sides of the heat source. In case of an acceleration, the gas moves to a direction that is opposite to that of the motion which results in a temperature rise in the thermopile that is nearer to the gas source. An output voltage is thus produced which is a measure of the acceleration [10].

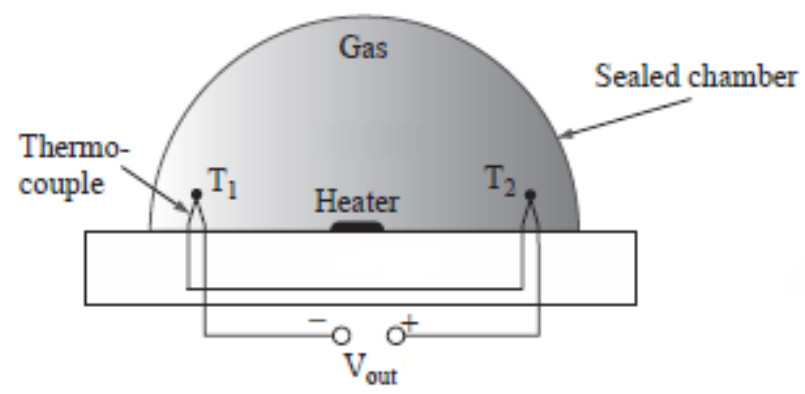

Figure 3.6: Heated gas accelerometer [10]

The accelerometers that are in use today are in the form of Microelectromechanical Systems (MEMS), miniature devices comprised of mechanical and electrical components. Early in their development, the mechanical parts (mass, beams, strain gauges, etc.) were produced separately, and the electrical components were placed externally. Nowadays, MEMS production allows easy production of accelerometers comprising of all of the components in a single package. MEMS based sensors provide a low cost and smaller size alternative to the traditional wire-based devices. Accelerometers are used for structural health monitoring purposes, for example, monitoring the state of bridges and other civil structures. A MEMS based accelerometer can also be easily incorporated in a transmission tower monitoring network. 
3.3 MEMSIC 2125 dual-axis accelerometer

The accelerometer used for the vibration detector is the MEMSIC2125

(MXD2125G/H/M/N). As shown in Figure 3.7, it is a low cost, dual axis accelerometer with a full-scale range of $\pm 3 \mathrm{~g}$ and a sensitivity of 12.5\%/g @5V at $25^{\circ} \mathrm{C}$ [22]. It can measure both dynamic acceleration (e.g. vibration) and static acceleration (e.g. gravity). Its high shock survival rating of 50,000 g ensures that it is not easily damaged by a shockwave, that would emanate from a lightning strike, which would be in the range of thousands of g's. It provides two digital outputs which produce pulses of 50\% duty cycle at zero g acceleration. The duty cycle of the output changes due to acceleration. The pin diagram and pin description are given in Figure 3.8 and Table 3.1 respectively. These were used later on for the circuit diagram of the system, in section 3.5 .

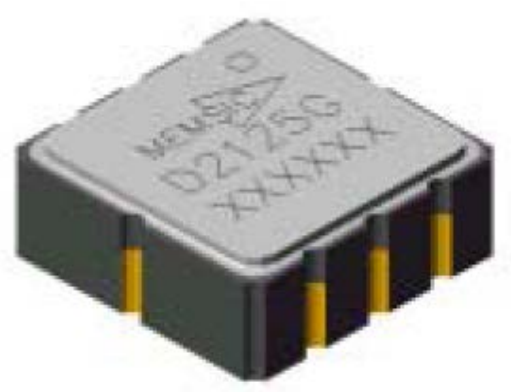

Figure 3.7: MEMSIC2125 accelerometer [22] 


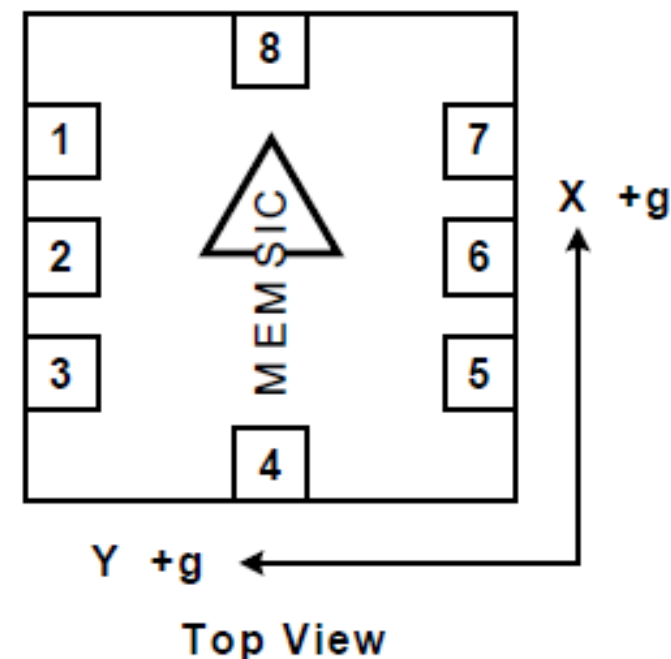

Figure 3.8: MEMSIC2125 pin diagram [22]

Table 3.1: Pin description of MEMSIC2125 [22]

\begin{tabular}{|l|l|l|}
\hline Pin & Name & Description \\
\hline 1 & Tout & Temperature (Analog voltage) \\
\hline 2 & Douty & Y-Axis acceleration digital signal \\
\hline 3 & Gnd & Ground \\
\hline 4 & VDA & Analog supply voltage \\
\hline 5 & Doutx & X-Axis acceleration digital signal \\
\hline 6 & Vref & 2.5V reference \\
\hline 7 & Sck & Optional external clock \\
\hline 8 & VDD & Digital supply voltage \\
\hline
\end{tabular}

The MEMSIC2125 accelerometer is a heated gas accelerometer with four thermopiles. At zero g acceleration, the temperature gradient is symmetrical about the heat source, thus the temperature is the same at all four thermopiles which causes them to produce the same output voltage. An acceleration experienced by the device will cause 
the temperature gradient to be asymmetrical, thereby resulting in the temperature and the voltage outputs of the thermopiles to be different. When the accelerometer is level, the hot gas pocket rises to the top center of the accelerometer's chamber and all the sensors measure the same temperature. If the accelerometer is tilted, the hot gases collect closer to one/two of the temperature sensors, as shown in Figure 3.9. The temperature measurements are translated as digital pulses which are easy to read with microcontrollers [22, 23].
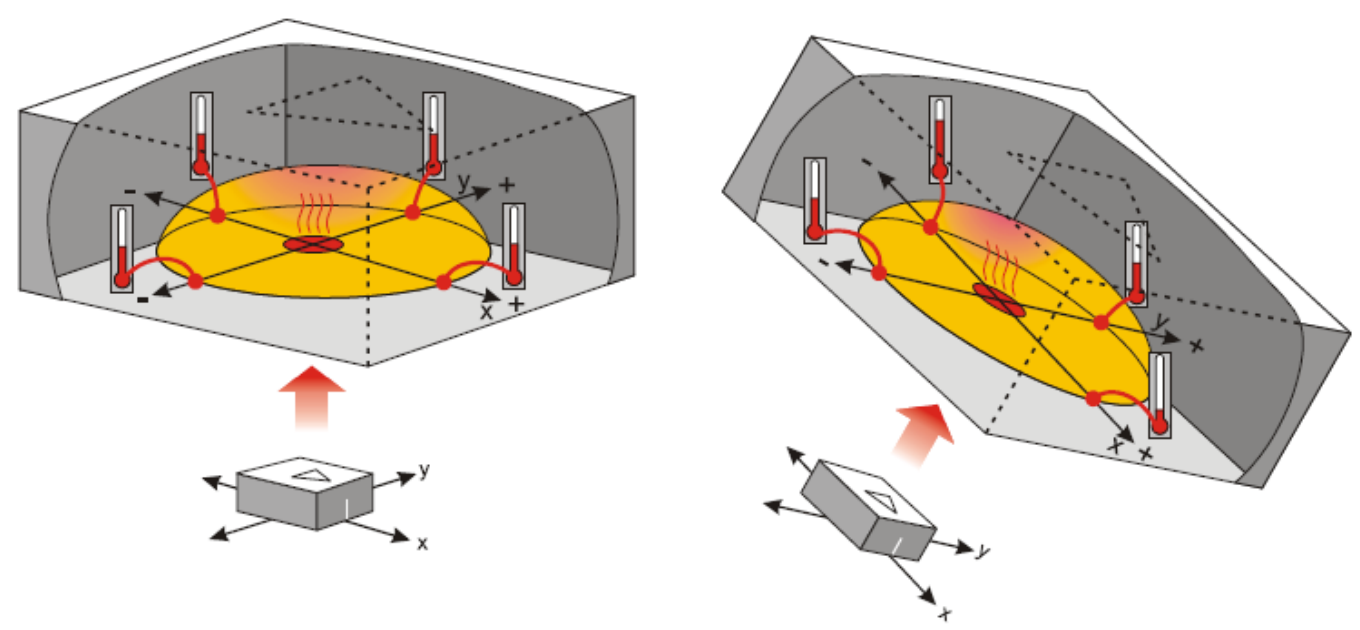

Figure 3.9: Inside view of the MEMSIC2125 [22]

\subsection{Microcontroller}

The Microcontroller used for reading data from the accelerometer and correlating that with the output from the current detector circuit was Arduino Uno. It is microcontroller board based on the ATMEGA328P microcontroller, as shown in Figure 3.10. It has 14 digital input/output pins, 6 analog input pins, a $16 \mathrm{MHz}$ quartz Crystal, a USB connection, a power jack, an (in-circuit serial programming) ICSP header and a reset button [24]. 


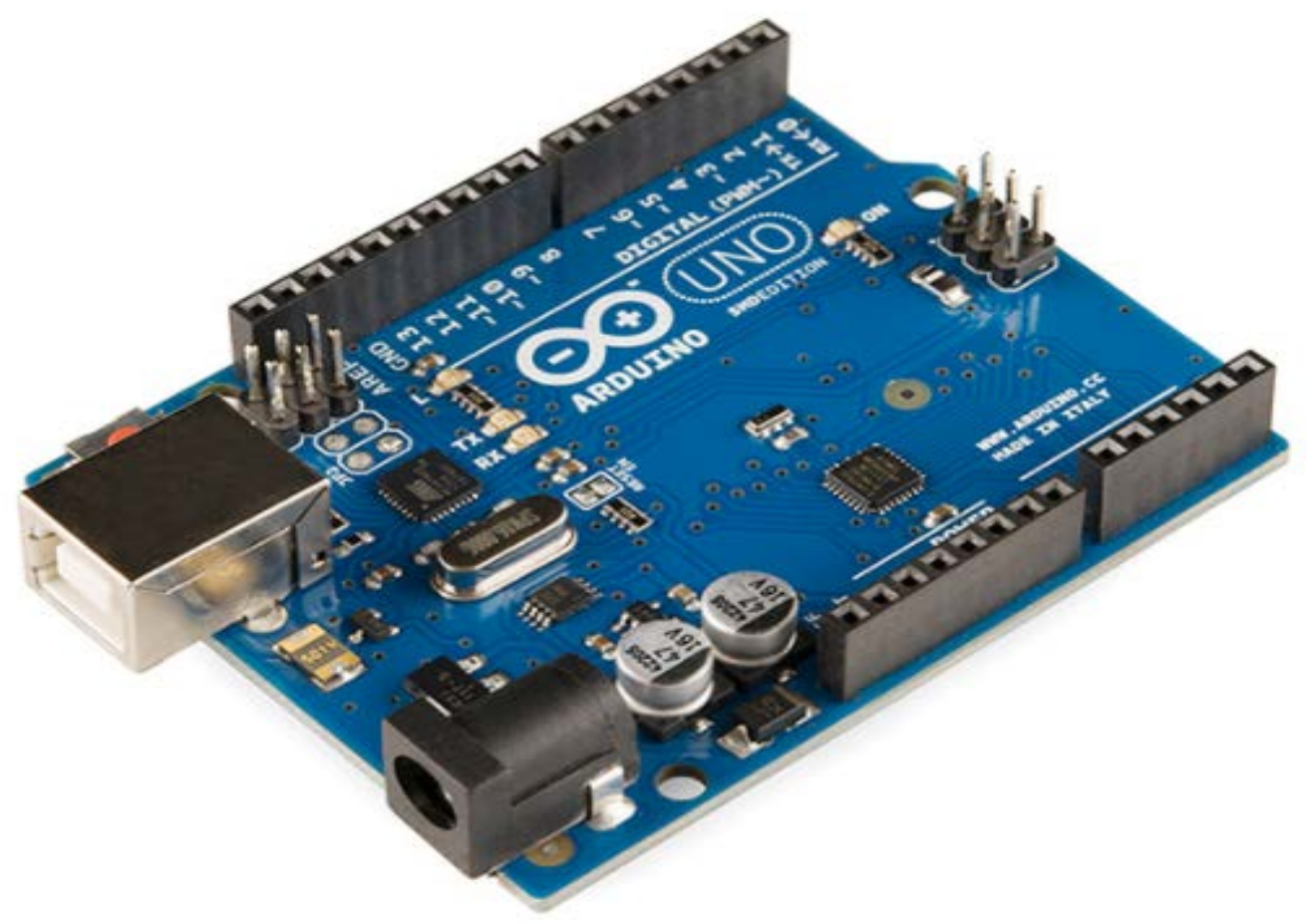

Figure 3.10: Arduino UNO microcontroller board [24]

\subsection{Proposed vibration detector}

Figure 3.12 shows the circuit diagram of the vibration detector. It was set up in a breadboard. The input power to the microcontroller was provided by the USB jack. It can also be powered by means of a $5 \mathrm{~V}$ adapter. The microcontroller was then used to provide input power to the digital supply voltage and analog supply voltage pins of the accelerometer. The capacitors are used to reduce the voltage ripple. The optional external clock of the accelerometer, pin 7, was grounded as it was not required for the operation. The $x$-axis and y-axis digital signal pins, pins 5 and 2 respectively, were connected to the PWM input pins of the microcontroller for reading the acceleration experienced by the accelerometer. The USB jack was connected to the computer to obtain the real time values of the accelerometer output by using the serial monitor in the Arduino IDE software. 


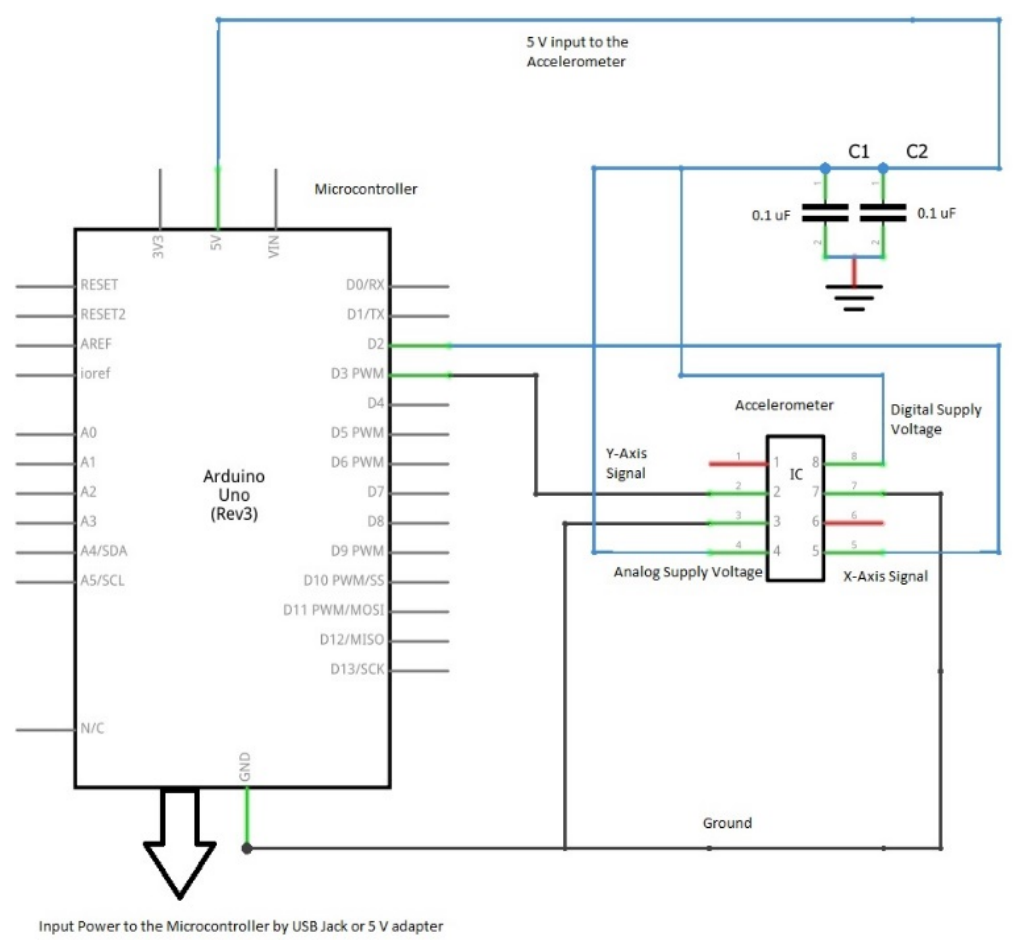

Figure 3.11: Circuit diagram of the vibration detector

Figure 3.13 shows the flowchart for vibration detection. After the initialization, a calibration step is put into place which was used to determine the maximum and minimum values of acceleration experienced in the $\mathrm{x}$ and $\mathrm{y}$ axis respectively, while in the rest position. An acceleration experienced in $\mathrm{x}$-axis (acc. $\mathrm{x}$ ) read by the microcontroller is incremented by 10 milli g’s to obtain max acc. $\mathrm{x}$ and decremented by 10 milli g's to obtain min acc. x. Similarly, a value of an acceleration experienced in y-axis (acc. y) is used to obtain max acc. y and min acc. y. If acc. $x$ and/or y-axis acc. y either exceeds the maximum acceleration or goes below the minimum acceleration, then vibration notification is sent by the vibration detector. 


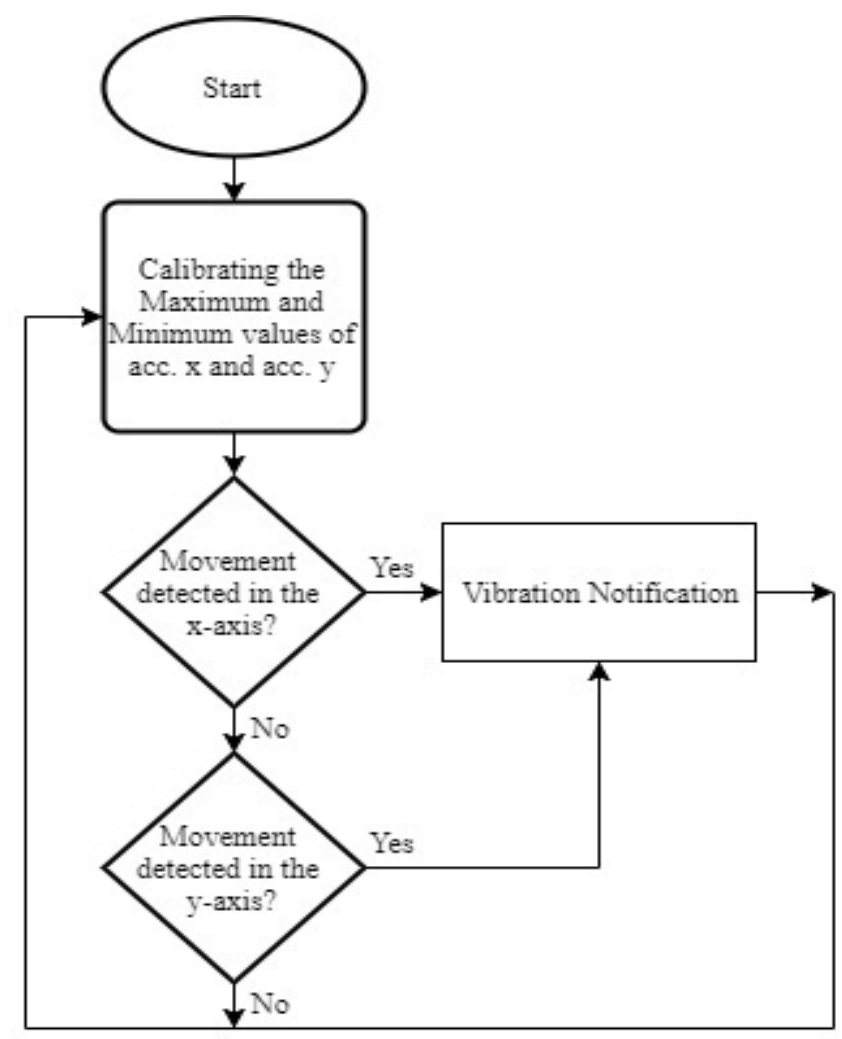

Figure 3.12: Flowchart for vibration detection

\subsection{Summary}

The workings of the vibration detector are discussed in this chapter with the aid of a block diagram, circuit diagram and a flowchart. This chapter discusses the vibration detector, and its various components, which is required to detect the vibration at the instant of the lightning strike. A basic overview is also given on the types of accelerometers that are commonly used for various purposes related to vibration sensing. In addition, the working principle of the dual-axis accelerometer used for the prototype is also discussed. The vibration detector will be combined with the current detector to make up the combined lightning strike detector system. 


\section{CHAPTER IV}

\section{COMBINED LIGHTNING STRIKE DETECTOR SYSTEM}

This chapter discusses the combined lightning strike detector system. The working principle of the lightning strike detector is described with the aid of a block diagram and a flowchart. The microcontroller introduced in Chapter 3 was used for combining the current detector and the vibration detector. A sketch is also used to elucidate the workings of the combined lightning strike detector system.

\subsection{Lightning strike detector system}

The block diagram of the lightning strike detector is given in Figure 4.1. The current detector discussed in Chapter 2 and the vibration detector discussed in Chapter 3 were unified using the microcontroller. The output of the current detector was connected to the analog read port A0 of the microcontroller. The microcontroller was connected to a computer using a USB connector. The output was then obtained using the serial monitor in the Arduino IDE software.

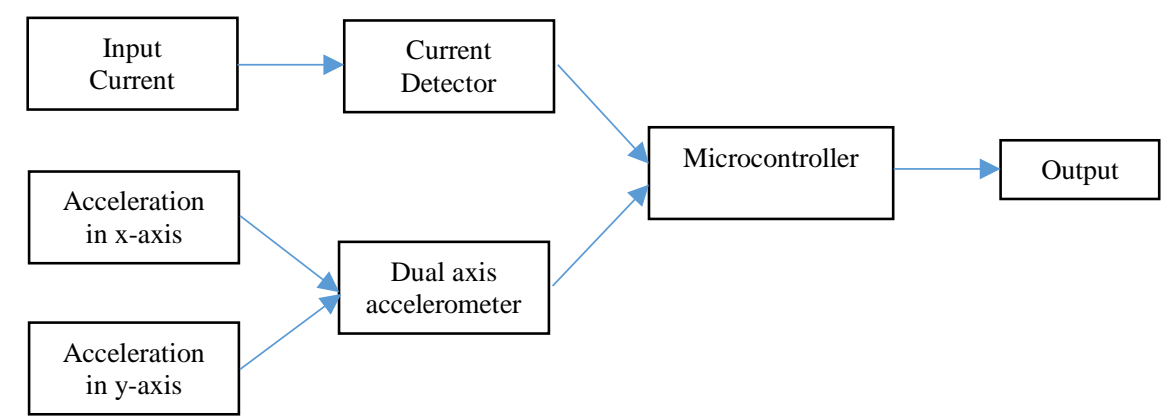

Figure 4.1: Block diagram of the lightning strike detector 
The flowchart of the lightning strike detector is given in Figure 4.2. A current detection notification is given if the output of current detector exceeds a threshold voltage of $0.5 \mathrm{~V}$. In case of only vibration being detected and the current detector not registering a notification, a vibration notification is sent. In case of only current being detected and the vibration detector not registering a notification, a current detection notification will be sent which signifies that even though lightning current was detected, the strike occurred elsewhere. In case of both vibration as well as the current being detected by the detector, a lightning strike detection notification will be sent which shows that a lightning strike occurred in the tower of interest.

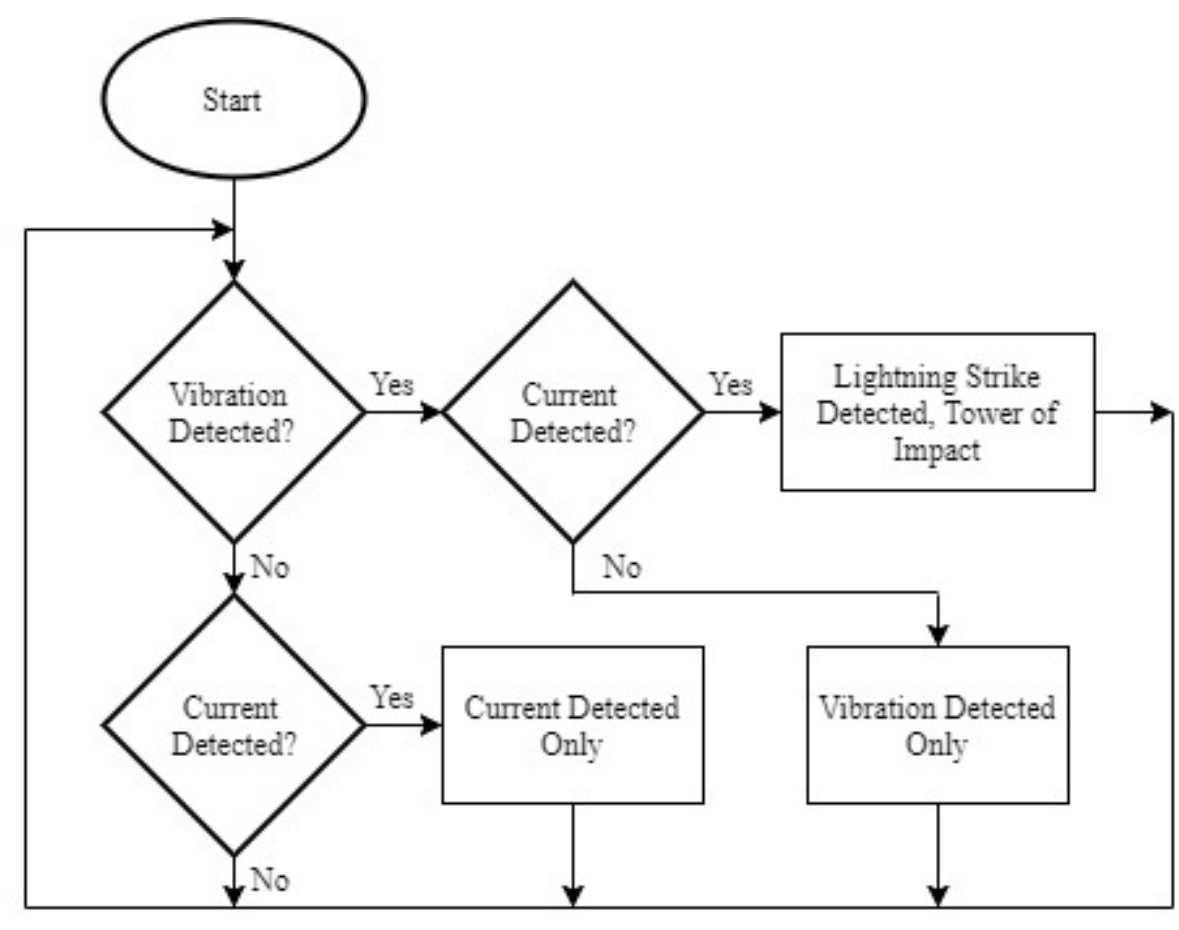

Figure 4.2: Flowchart for the lightning strike detector

Figure 4.3 shows a rough sketch of the lightning strike detector output, plotted using arbitrary data, and how vibrations due to various causes are distinguished. A vibration not accompanied by a current being detected by the current detector, i.e. a 
vibration notification is given by the vibration detector whereas a current detection notification is not given by the current detector, is disregarded as it could be due to other causes such as high wind speed or strikes to a distant tower. A vibration, being detected after a time delay of $\Delta \mathrm{t}$ from the strike, is accompanied by a current (the current detector notification is given by the current detector) and is thus notified as a vibration due to a lightning strike.

\section{Accelerometer Output}

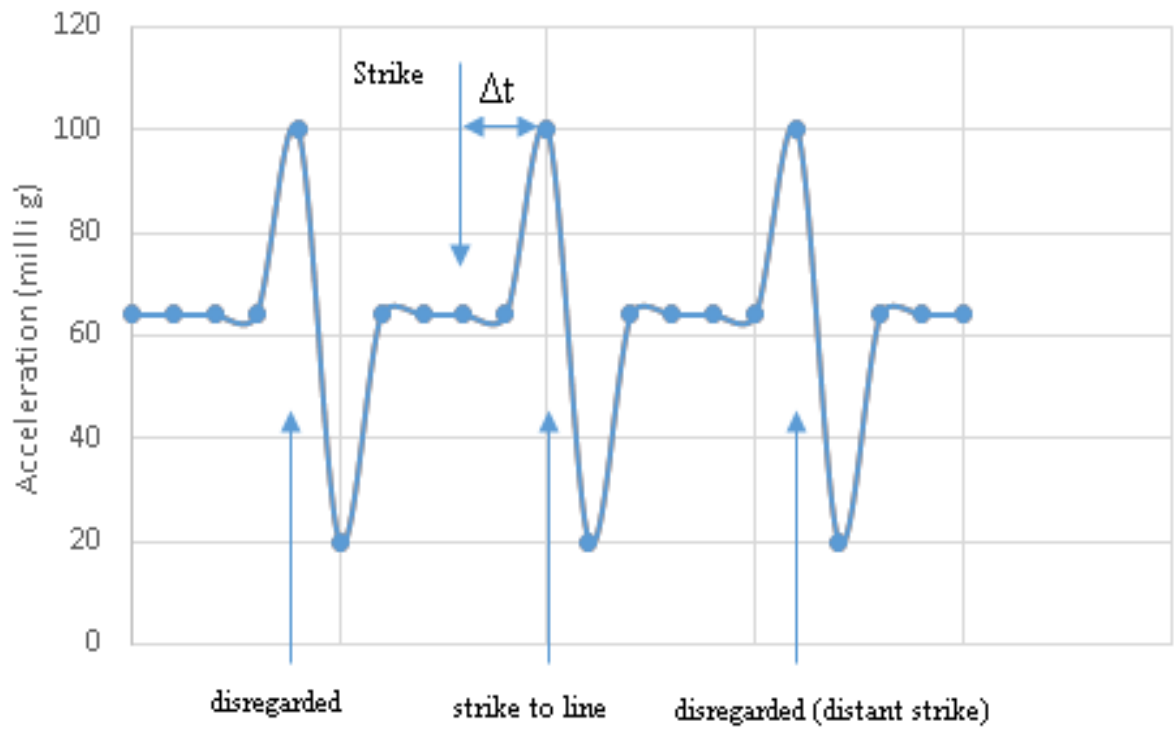

Current Detector Output

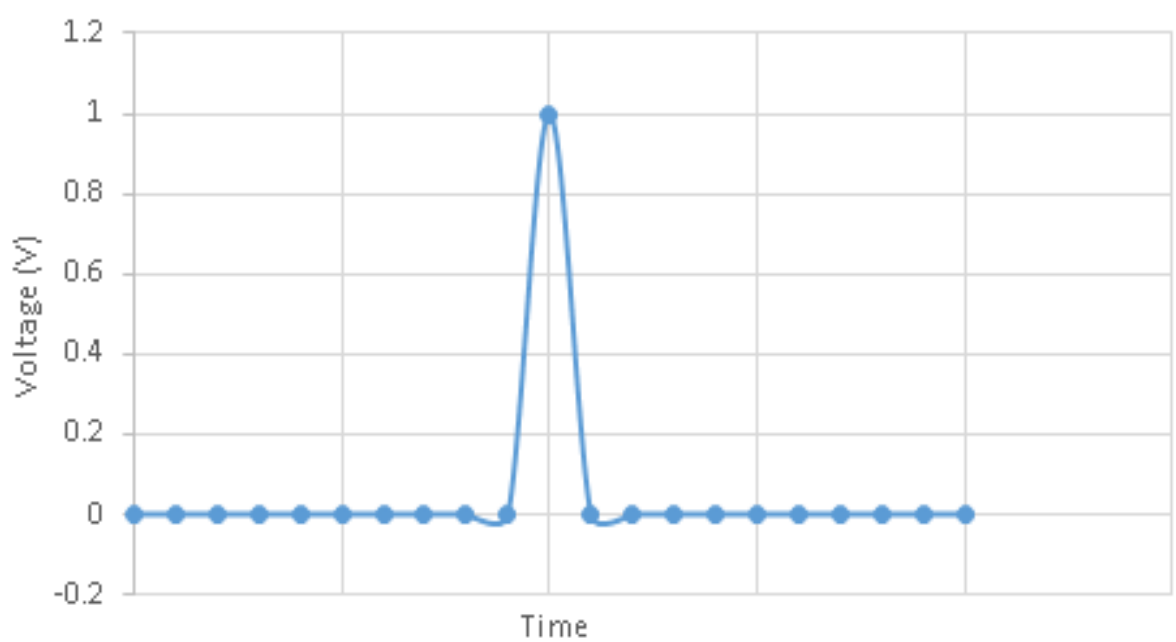

Figure 4.3: Sketch of the lightning strike detector output 


\subsection{Summary}

The workings of the combined lightning strike detector system are described in this section. A block diagram is used to show how the current detector and the vibration detector were unified to form the combined system. The flowchart shows how the combined system was programmed to respond to the vibration and current readings. A sketch is then used for further description of the combined system. 


\section{CHAPTER V}

\section{RESULTS FOR THE LIGHTNING STRIKE DETECTOR}

This chapter discusses the results of the lightning strike detector. The experimental results of the current detector are compared to the simulation results for validation. The serial monitor results for the vibration detector which features the acceleration values obtained in the $\mathrm{x}$ and $\mathrm{y}$-axis respectively provide a vibration notification in case a significant movement is detected. The results for the combined lightning strike detector show how the prototype responds to changing vibration and input current readings.

\subsection{Simulation results for the current detector}

The simulation results of the current detector are given in this section. Frequency response of the current detector was obtained using the transfer function of the current detector in MATLAB. Figure 5.1 shows the frequency response of the current detector. Lightning currents can have any frequency in the range of $2 \mathrm{kHz}$ to $2 \mathrm{MHz}$. The current detector has a smooth response from $1 \mathrm{kHz}$ to $9.57 \mathrm{MHz}$, thus making it suitable for detecting lightning currents of various frequency ranges.

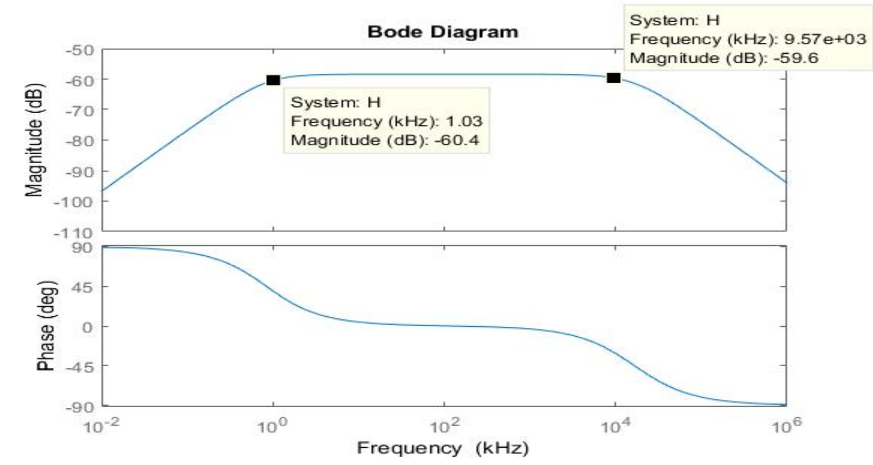

Figure 5.1: Frequency response of the current detector 
The simulation of the current detector was then carried out in LTspice. Sinusoidal currents and transient currents were used as inputs. A damped sinusoidal input current with an amplitude in the range of thousands of kilo amperes was used as the input in order to mimic a typical lightning current $[4,18,21$, and 25]. Figure 5.2 shows the frequency response of the current detector from the LTspice simulation. The magnitude of the frequency response is flat between $1 \mathrm{kHz}$ and $2 \mathrm{MHz}$. As the lightning current frequency is also near the range where the frequency response is flat, the current detector can be used for the detection of lightning current. The magnitude of the frequency response is $-116 \mathrm{~dB}$, which is almost two times the $-60 \mathrm{~dB}$ magnitude of the frequency response obtained in the MATLAB simulation. The frequency response was also better for a higher range in case of the MATLAB simulation ( $1 \mathrm{kHz}$ to $9.57 \mathrm{MHz}$ ) compared to the LTspice simulation (1 kHz to $2 \mathrm{MHz}$ ). The differences could be attributed to the non-ideal nature of the components which was not taken into account when evaluating the frequency response in MATLAB.

The simulation was initially carried out using a high input current with maximum peak amplitude of $100 \mathrm{kA}$. The frequency was chosen to be $100 \mathrm{kHz}$ as the mean frequency of the lightning current is around $100 \mathrm{kHz}$ and is the standard lightning current frequency as given in IEC 62305 lightning protection standard [26]. This simulation was done for observing the behavior of the Rogowski coil in presence of current in the range of kA's which was later compared with the behavior of the Rogowski coil in presence of current in the range of mA's. The constant sinusoidal input current is given in Figure 5.3. The induced emf across the Rogowski coil and the output voltage are given in Figures 5.4 and 5.5 respectively. After an initial transient period of $0.2 \mathrm{~s}$, the induced emf of the Rogowski coil follows the input fairly well. As the transient was not considered important, the time axis of Figure 5.5 was chosen to 
be much higher than the axes of Figures 5.3 and 5.4 to allow time for the output voltage to reach a steady state value of $4.6 \mathrm{~V}$. These results show that the Rogowski coil can be used for the detection of a high frequency sinusoidal current in the range of kA's.

The simulation was then carried out using a damped sinusoidal current for observing the transient behavior of the Rogowski coil in presence of current in the range of kA's. The damped sinusoidal input current is given in Figure 5.6. The induced emf across the coil is given in Figure 5.7. Figure 5.7 in particular shows the linear response of the coil as the output follows the input fairly well. Figure 5.8 shows the output voltage of the current detector with the damped sinusoidal current. Due to the lack of a constant steady supply of current as in Figure 5.5, the output voltage of Figure 5.8 falls rapidly as soon as the transient current reaches zero. These results show that the Rogowski coil can be used to detect a damped sinusoidal current in the range of kA's.

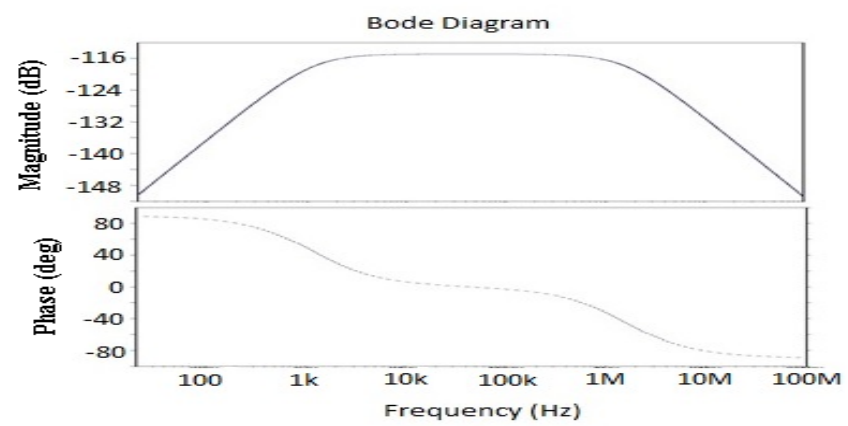

Figure 5.2: Frequency response of the current detector (circuit simulation) 


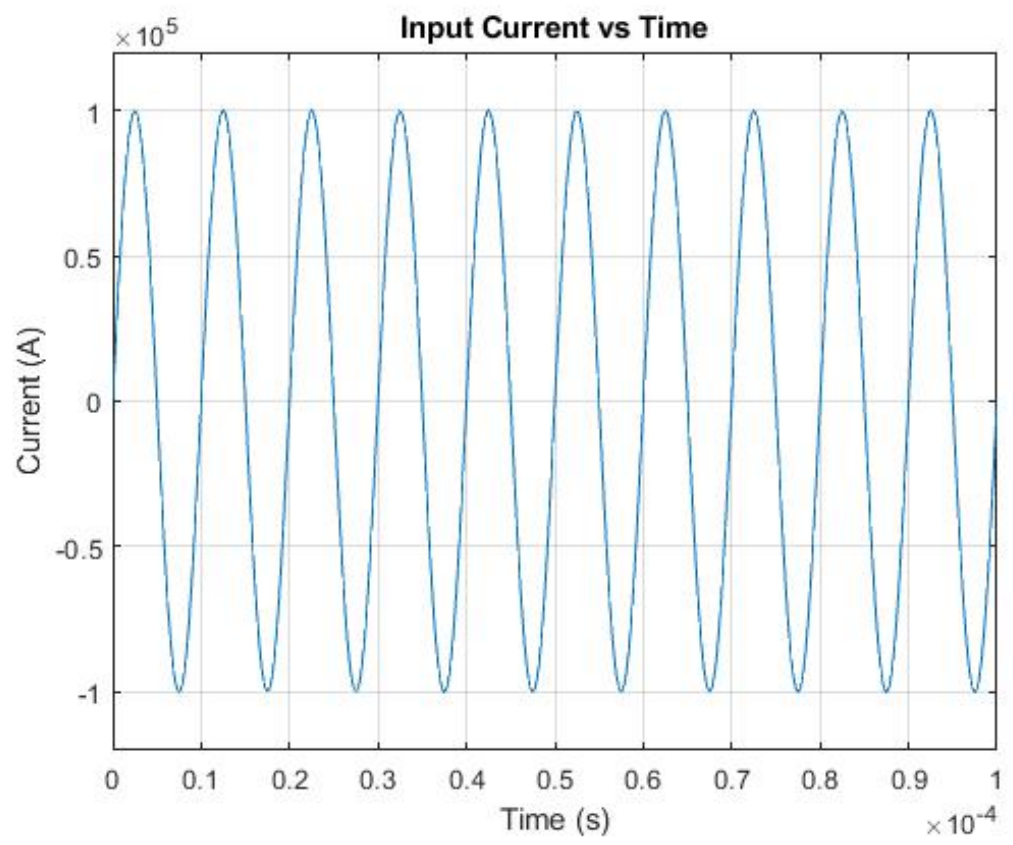

Figure 5.3: Simulated sinusoidal input current

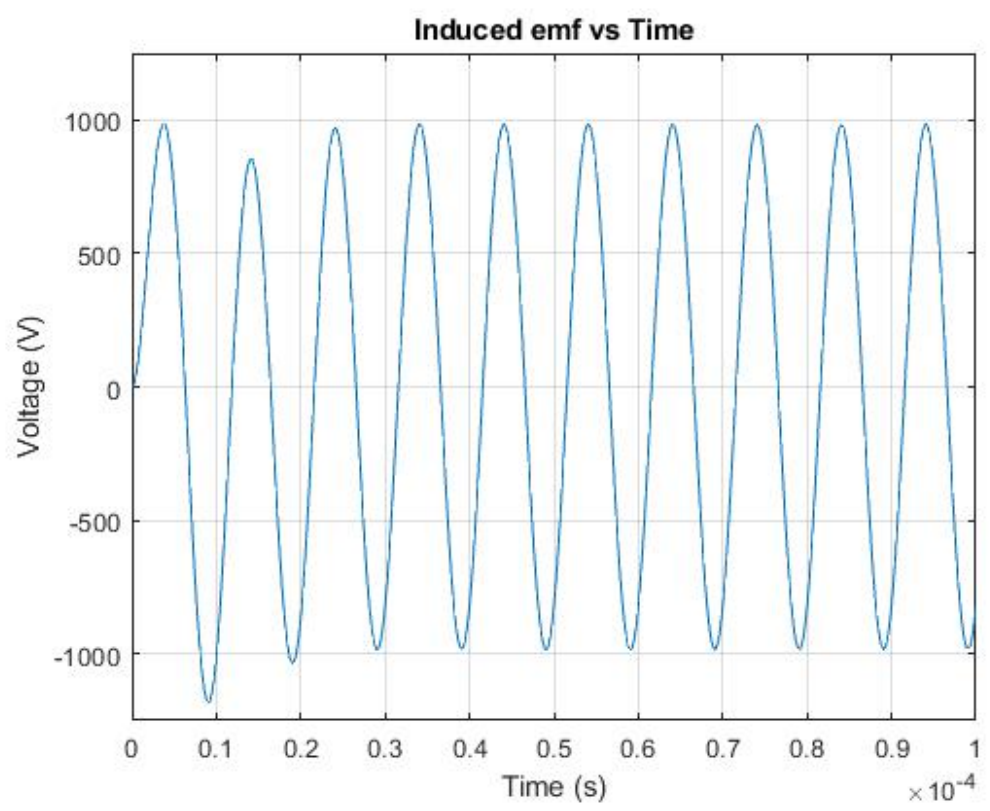

Figure 5.4: Induced emf across the Rogowski coil with sinusoidal input current 


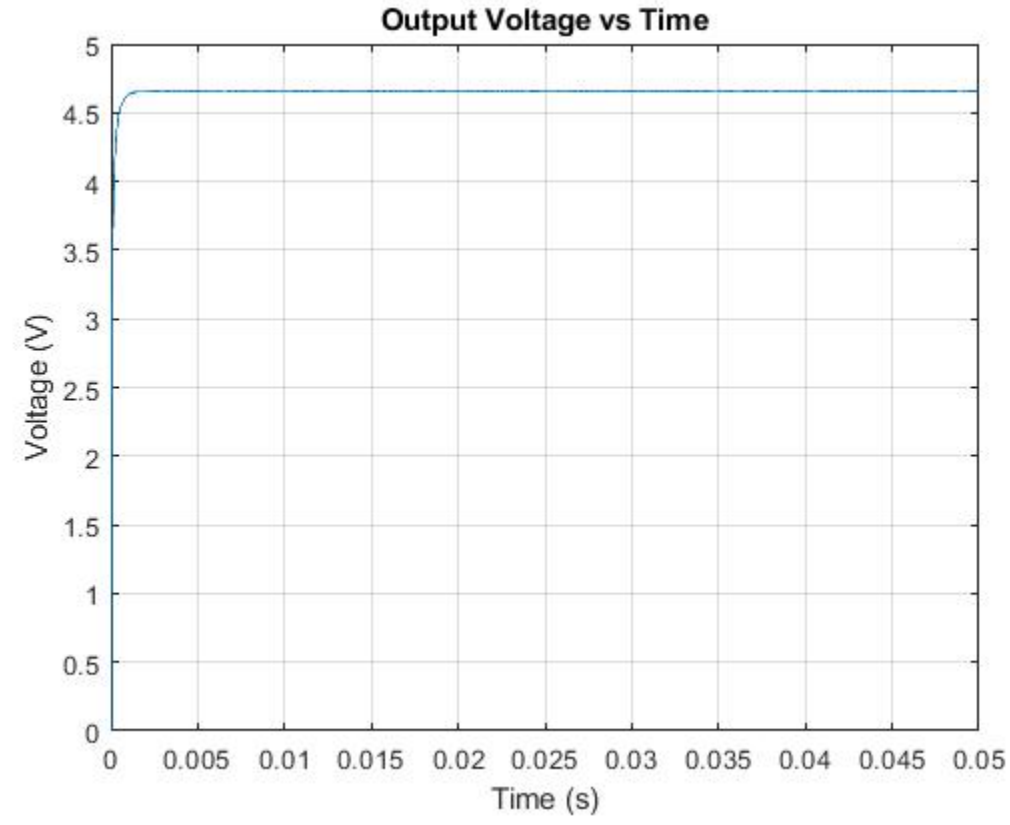

Figure 5.5: Output voltage of the current detector with sinusoidal current

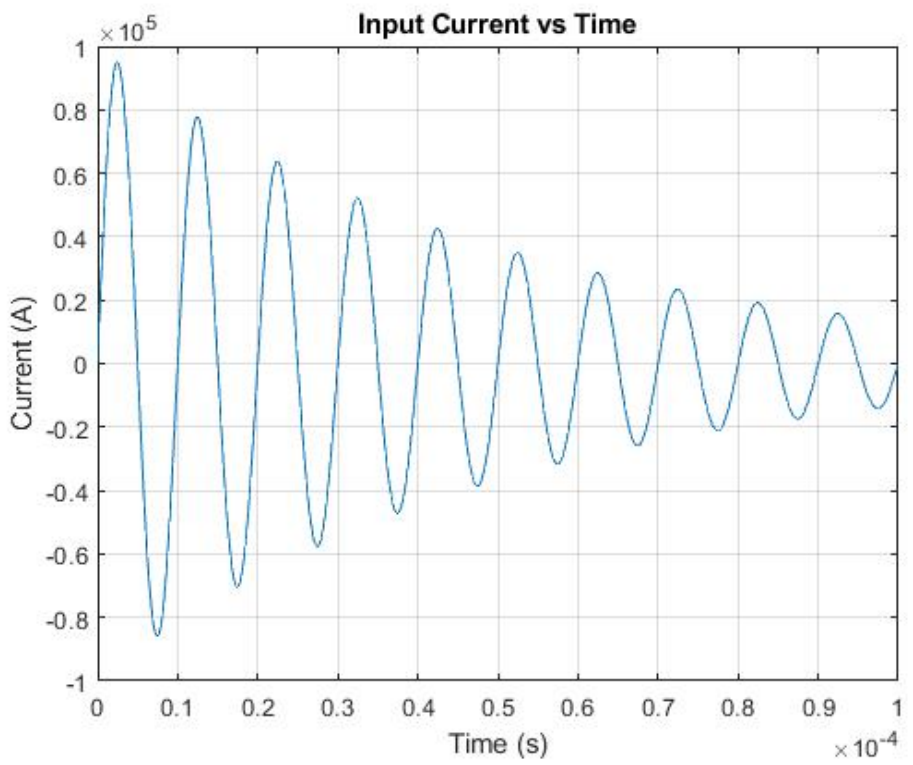

Figure 5.6: Simulated damped sinusoidal current 


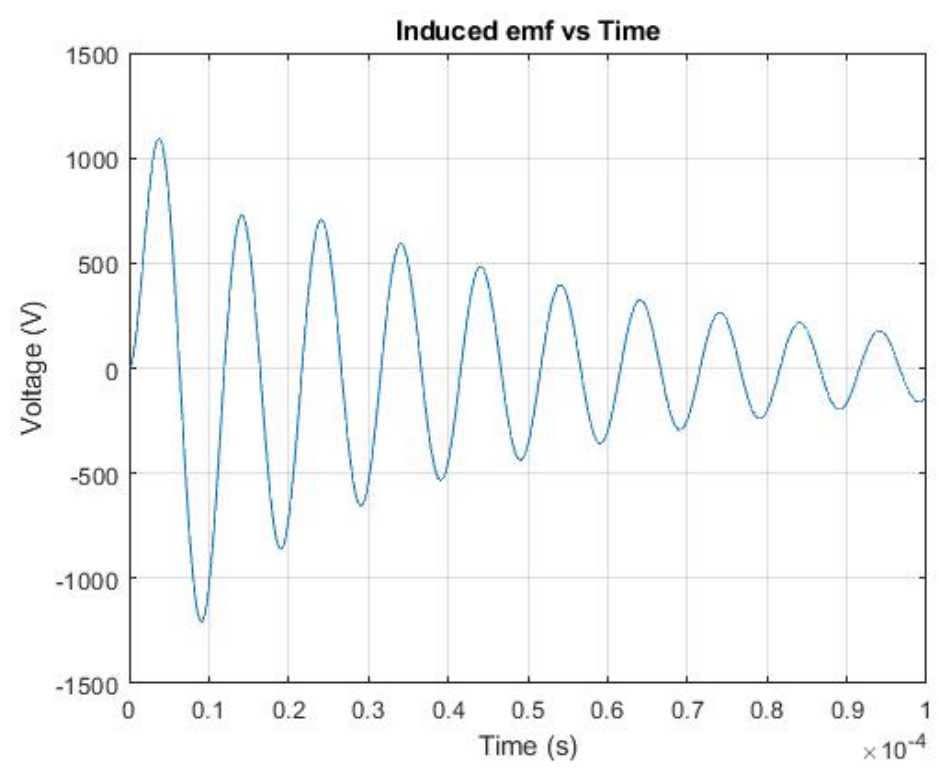

Figure 5.7: Induced emf across the Rogowski coil with damped sinusoidal current

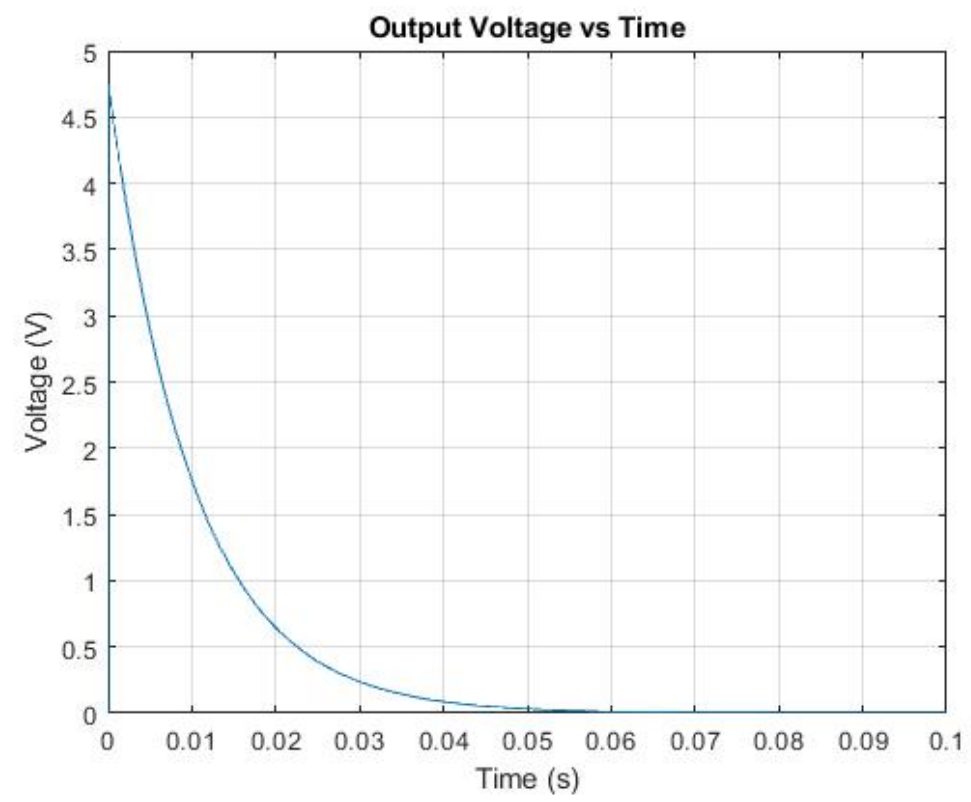

Figure 5.8: Output voltage with the damped sinusoidal current

The simulation was then carried out using a small input current with maximum peak amplitude of $300 \mathrm{~mA}$. The sinusoidal input current is given in Figure 5.9. The induced emf across the Rogowski coil and the output voltage are given in Figures 5.10 and 5.11 respectively. The damped sinusoidal current is given in Figure 5.12. The induced emf across the coil is given in Figure 5.13. Figure 5.14 shows the output 
voltage with the damped sinusoidal current. The simulation results show that the behavior of the Rogowski coil is similar for both high and low currents. As it is not possible to do a large scale test due to the unavailability of instruments, for practical purpose, a small current can be used for proper testing of the Rogowski coil.

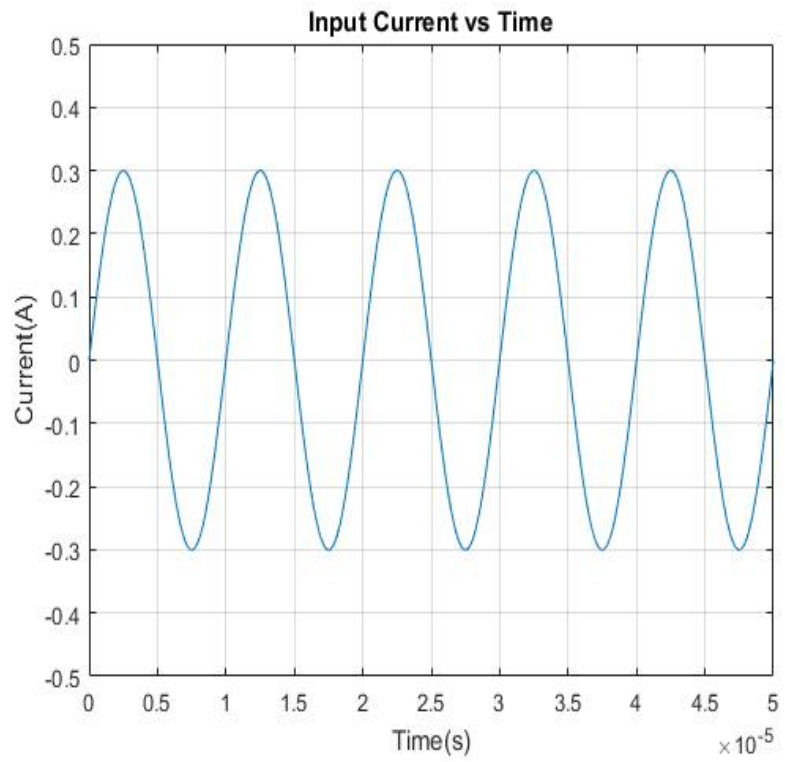

Figure 5.9: Simulated sinusoidal input current

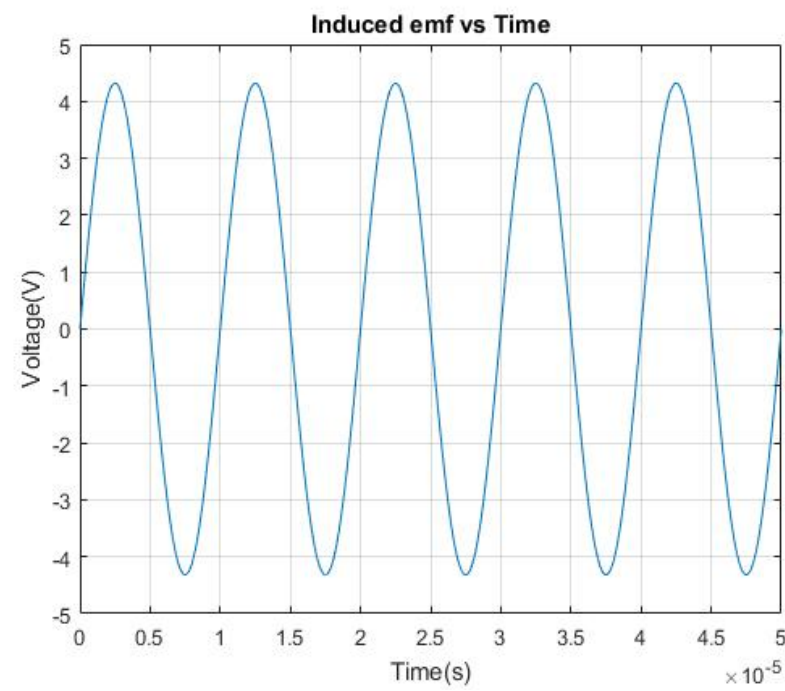

Figure 5.10: Induced emf across the Rogowski coil with sinusoidal input current 


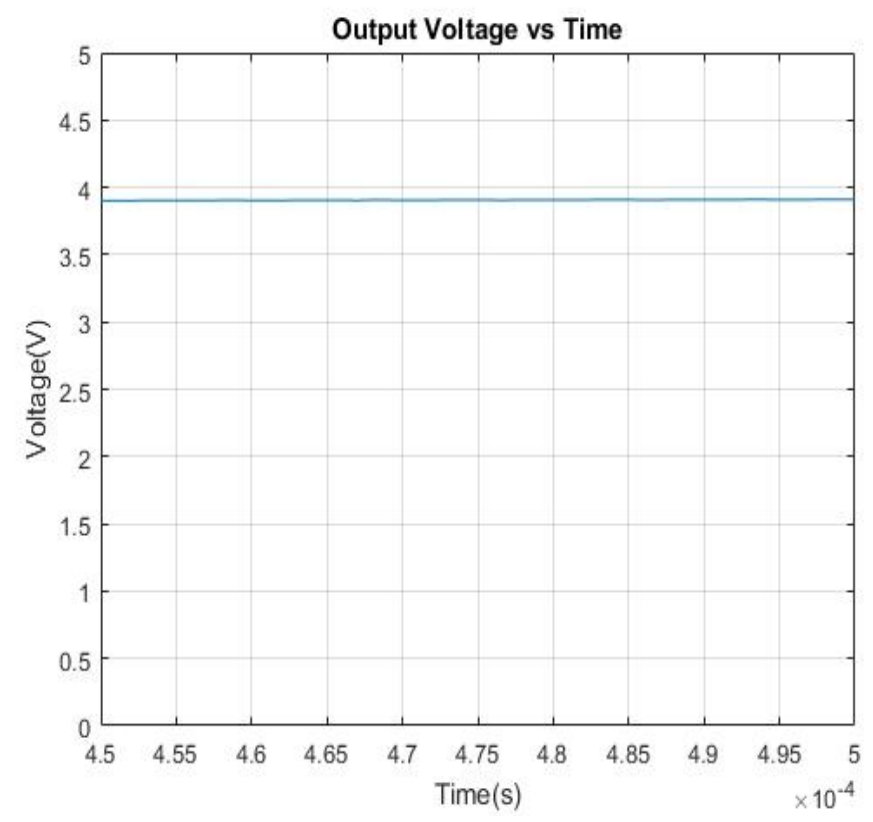

Figure 5.11: Output voltage of the current detector with sinusoidal current

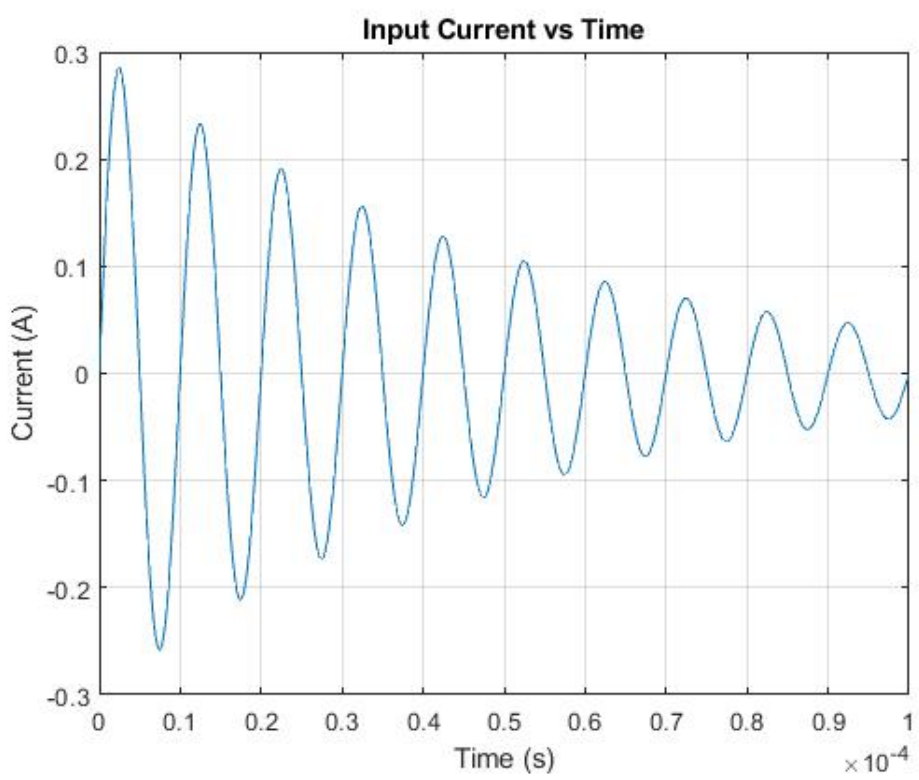

Figure 5.12: Simulated damped sinusoidal current 


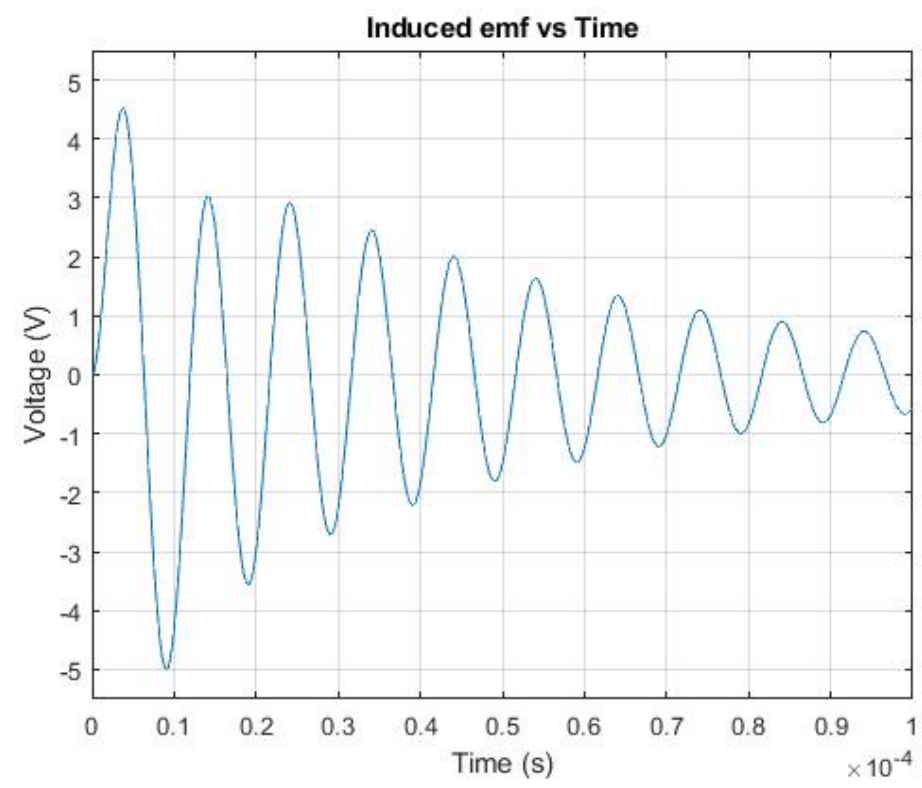

Figure 5.13: Induced emf across the Rogowski coil

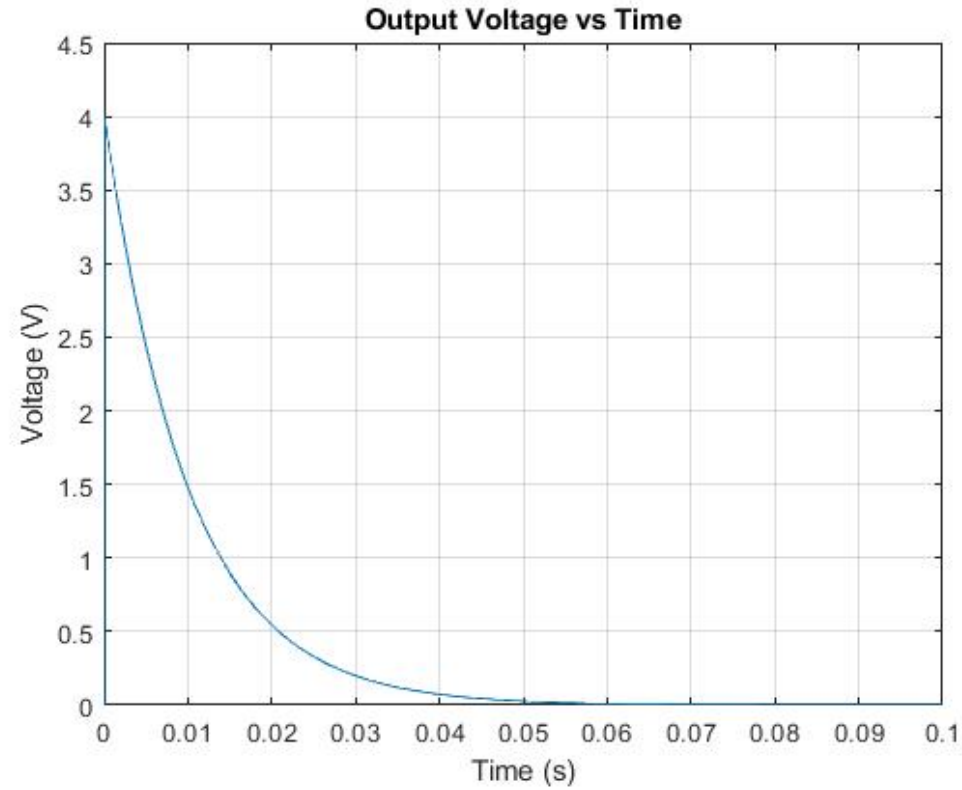

Figure 5.14: Output voltage with the damped sinusoidal current

Experimentally, it was not possible to produce a transient current which would last for 100-200 us. Thus, a transient current of 50 ms pulse duration, as shown in Figure 5.15 was simulated to obtain the output in Figure 5.16 for valid comparison with the experimental data. 


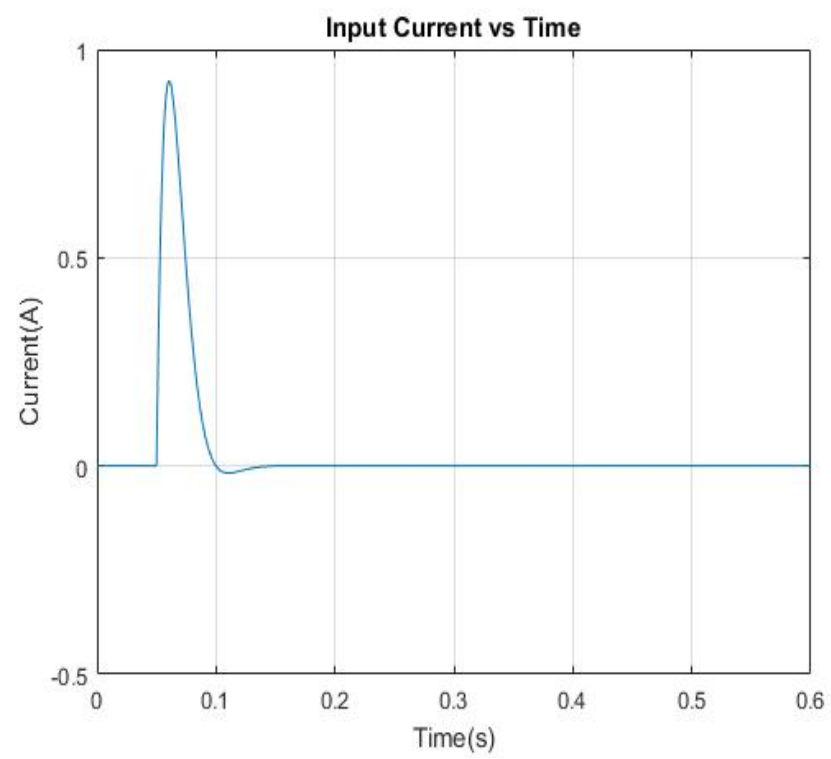

Figure 5.15: Simulated transient current

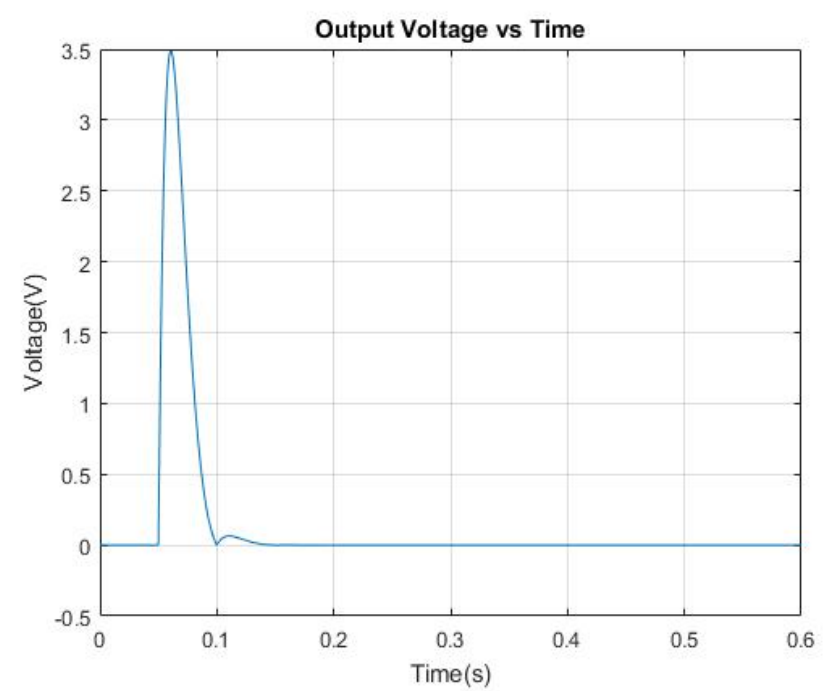

Figure 5.16: Output voltage with the transient current

5.2 Experimental results for the current detector

Figure 5.17 shows the experimental setup of the current detector as well as the lightning strike detector prototype. The Rogoswki coil was placed around a connecting wire in order to detect the current passing through the wire and its output connected to the rest of the current detector circuit on a breadboard. A signal 
generator was used as a source of current of peak magnitude $300 \mathrm{~mA}$ and $100 \mathrm{kHz}$ frequency to test the prototype for sinusoidal current. It was used to pass current through the connecting wire. A DC voltage source was used as the transient current generator by passing current through the connecting wire to test the prototype for transient current.

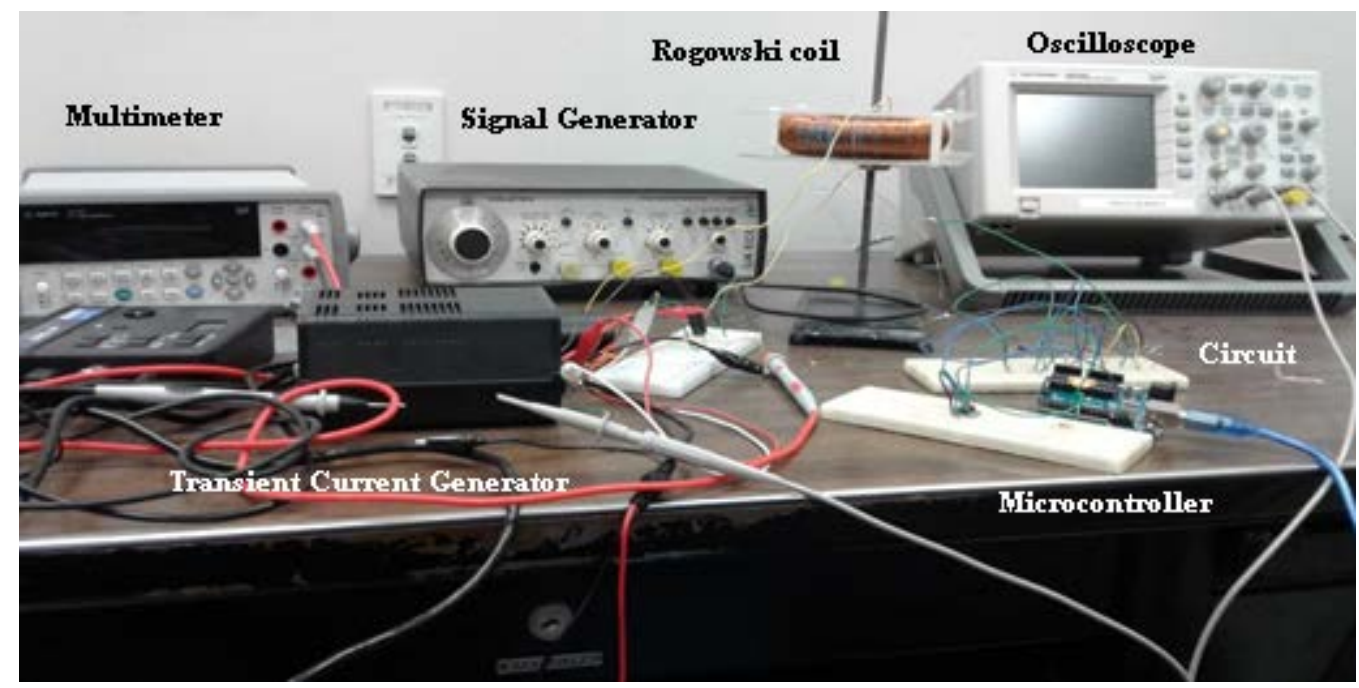

Figure 5.17: Experimental setup

Figure 5.18 shows the number of turns vs. emf of the coil whereas Figure 5.19 shows the input current against the output voltage of the coil. Linear graphs were obtained in both cases thus verifying the theoretical formulas of the Rogowski coil especially the formula for the induced emf in the coil given by (3) in section 2.2. A linear graph was obtained in Figure 5.18 which satisfies (3) where the no. of turns is linearly proportional to the emf, provided the other parameters are kept constant. Similarly, in Figure 5.19, the linear graph indicates that the input current is proportional to the induced emf and hence, proportional to the output voltage as long as the other parameters are kept constant. This shows that the experimental results conformed to the theoretical formulas. 


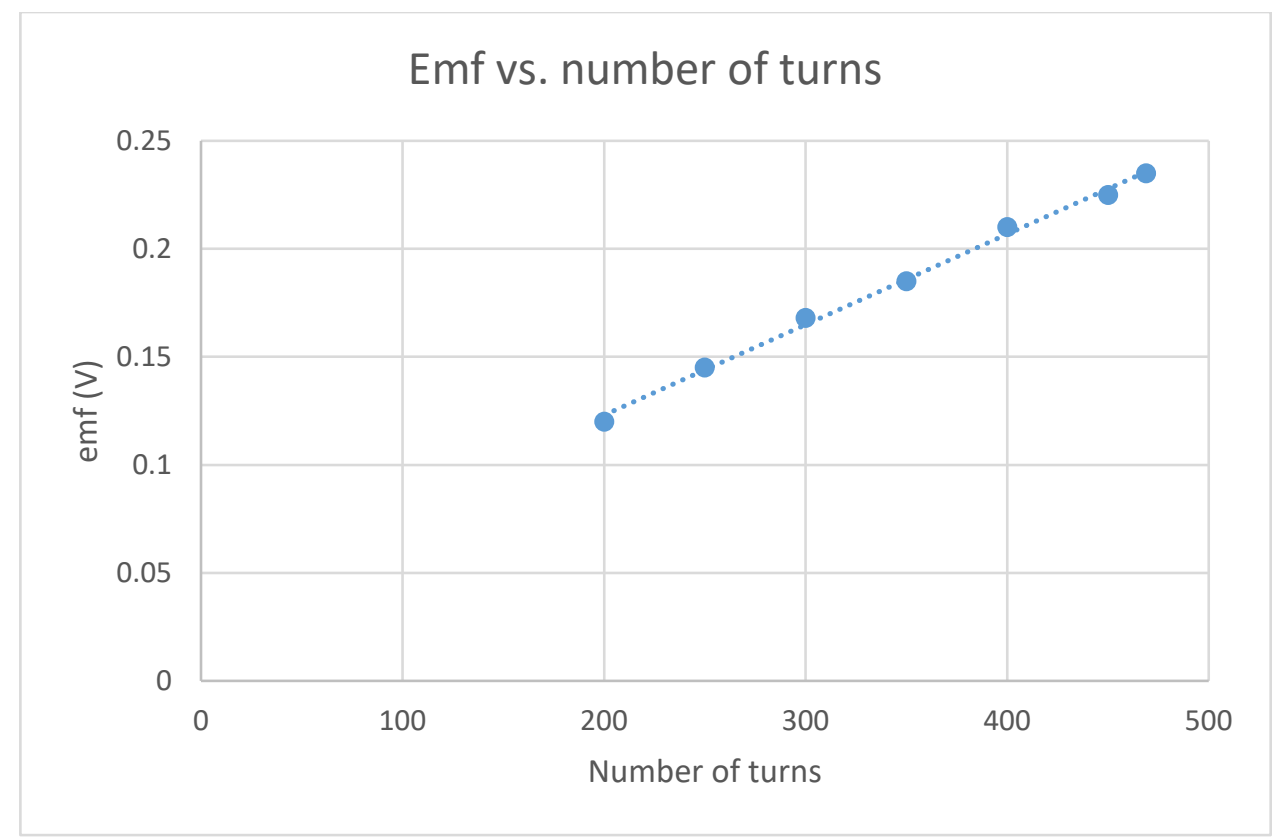

Figure 5.18: Plot of the induced emf (y-axis) against no. of turns of the coil (x-axis)

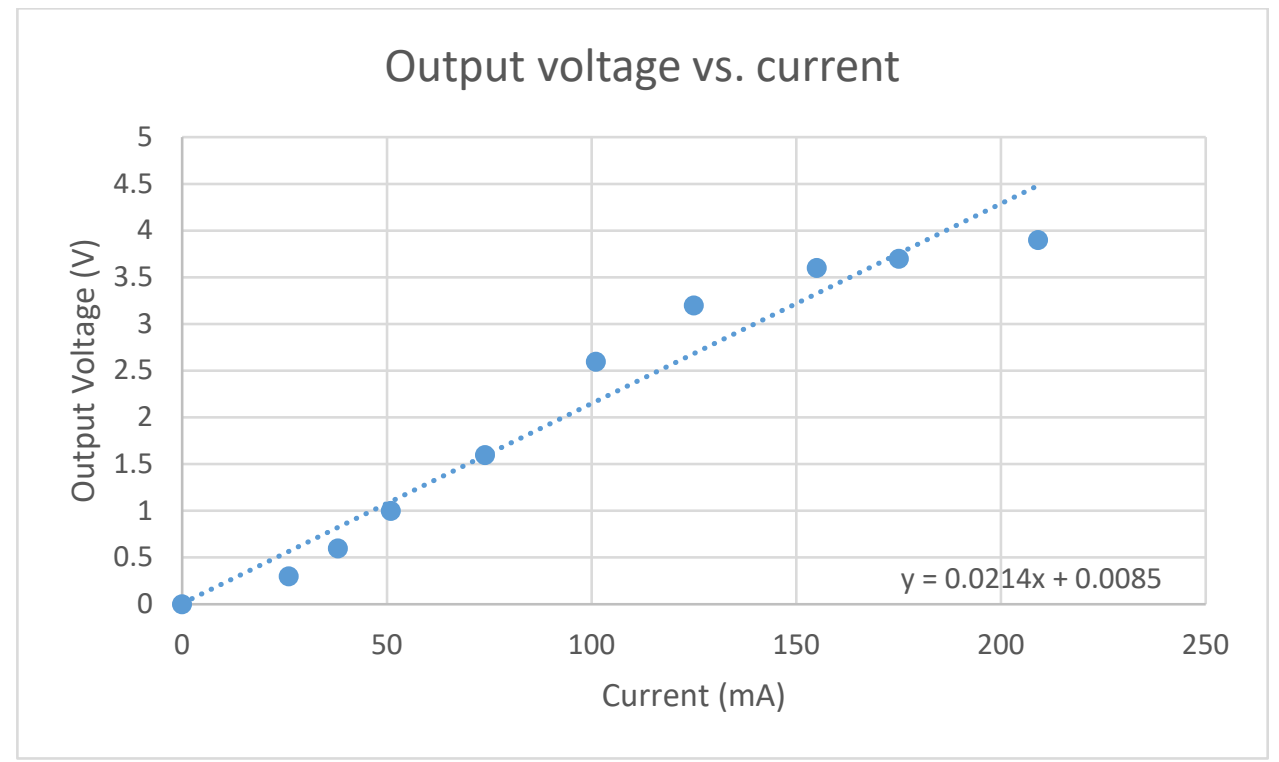

Figure 5.19: Plot of the output voltage (y-axis) against current I (x-axis)

Figure 5.20 shows the constant sinusoidal current used as input to the current detector for both the experimental setup and the simulation. The induced emf of the coil and the output of the current detector are given in Figures 5.21 and 5.22 respectively. A side by side comparison between the experimental and simulation results are given in both cases. The output waveforms from both the simulation and 
experimental results are similar in shape. The induced emf of the Rogowski coil was a sinusoidal voltage with $4.0 \mathrm{~V}$ peak magnitude and $100 \mathrm{kHz}$ frequency whereas the induced emf of the Rogowski coil in the simulation was a sinusoidal voltage with 4.3 V peak magnitude and $100 \mathrm{kHz}$ frequency. The current detector in the prototype produced $3.6 \mathrm{~V}$ whereas the current detector in the simulation produced $3.9 \mathrm{~V}$.
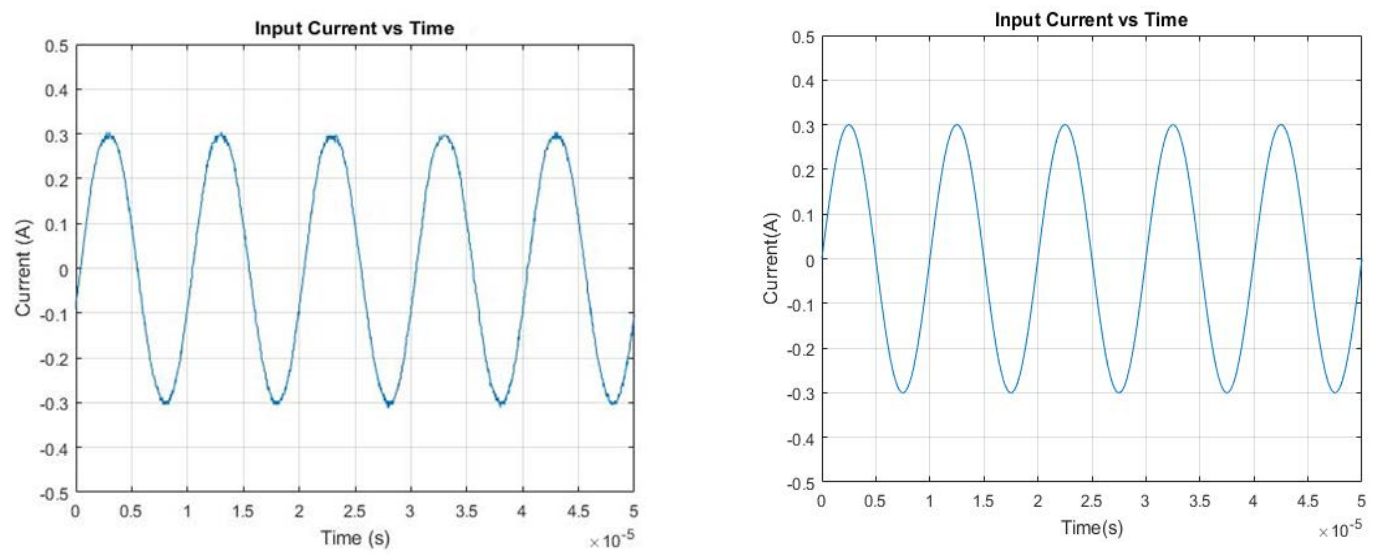

Figure 5.20: The constant sinusoidal input current used in the experimental setup (left) and the simulation (right)
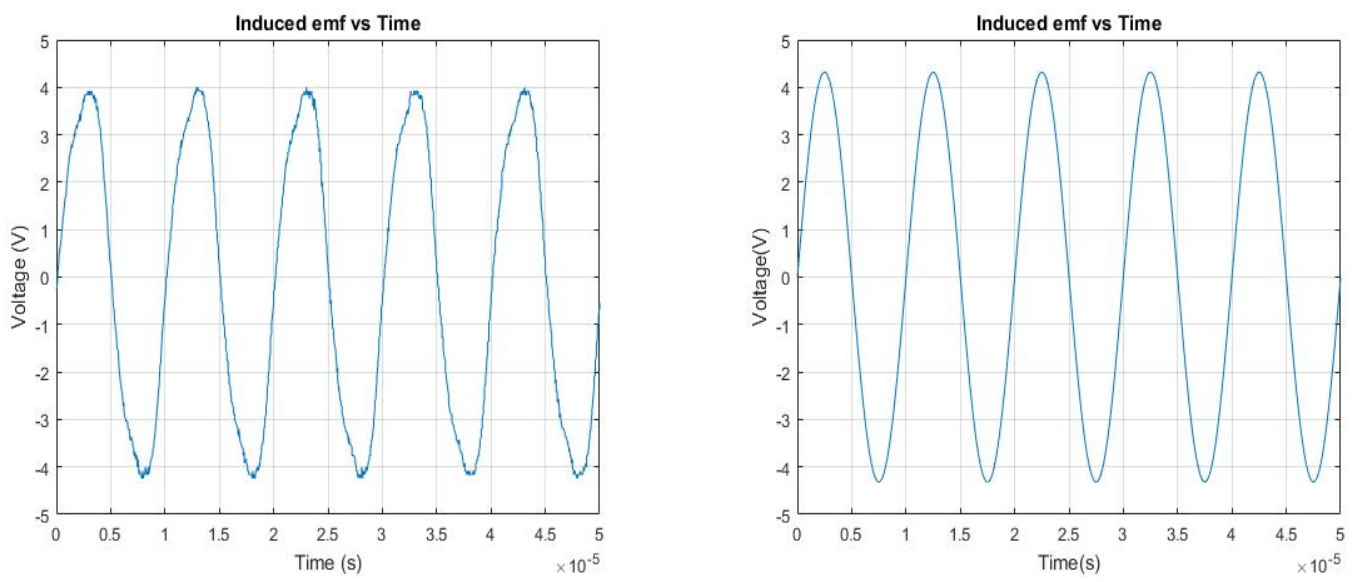

Figure 5.21: Induced emf across the Rogowski coil with sinusoidal input current for the experimental setup (left) and the simulation (right) 

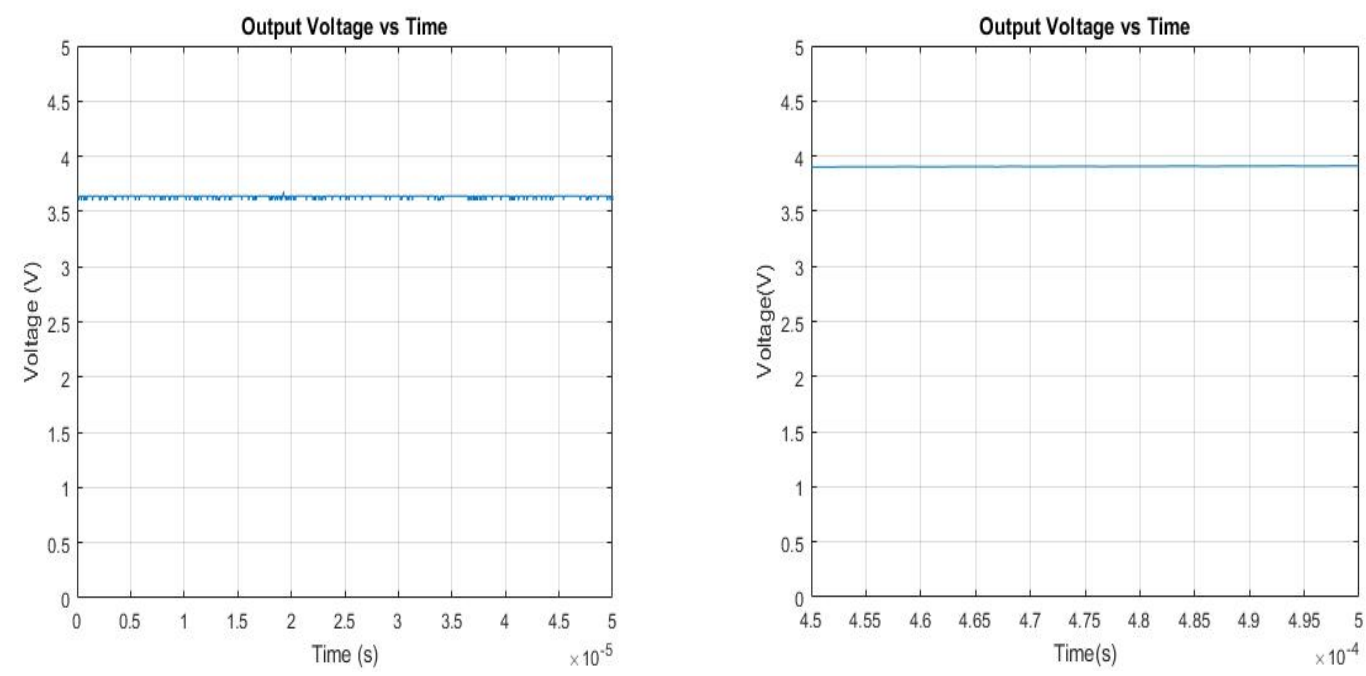

Figure 5.22: Output of the current detector with sinusoidal input current for the experimental setup (left) and the simulation (right)

Figure 5.23 shows the transient current waveform used as input to the current detector for both the experimental setup and the simulation. Figure 5.24 shows a side by side comparison between the experimental output of the current detector and the simulation output. The output waveforms from both the simulation and experimental results are similar in shape. The current detector in the prototype produced $3 \mathrm{~V}$ with a transient current of 0.9 A peak amplitude and 50 ms pulse duration whereas the current detector in the simulation produced 3.5 V. The discrepancies could be due to skin effect, which causes the resistance of the coil to be increased at higher frequencies, a phenomenon absent in the case of simulation. The differences (less than 10\%) could also be attributed to the non-ideal behavior of the components in the prototype. 

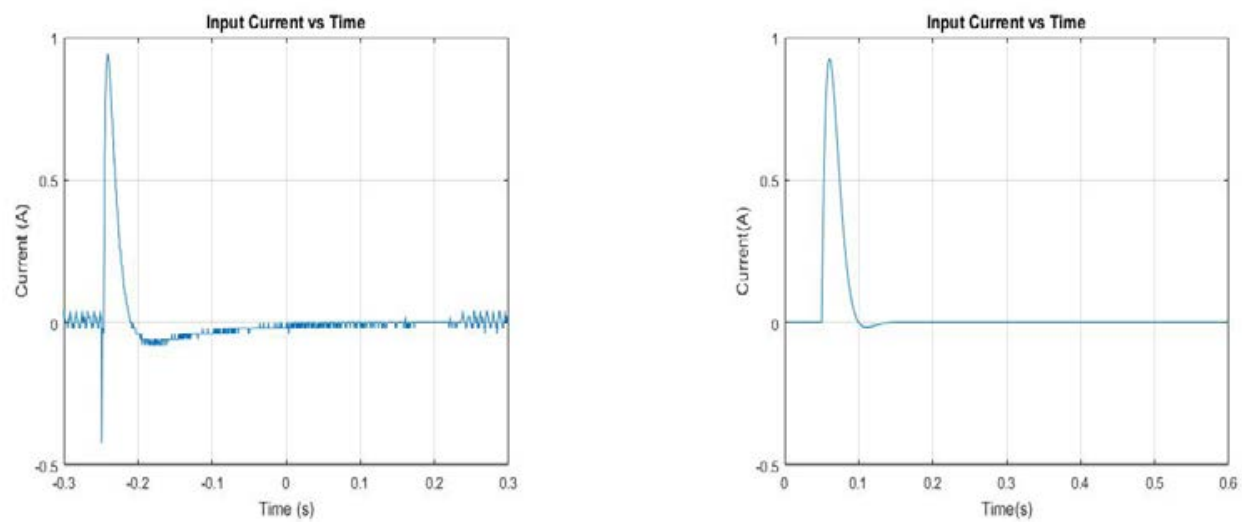

Figure 5.23: Transient input current for the experimental setup (left) and the simulation (right)
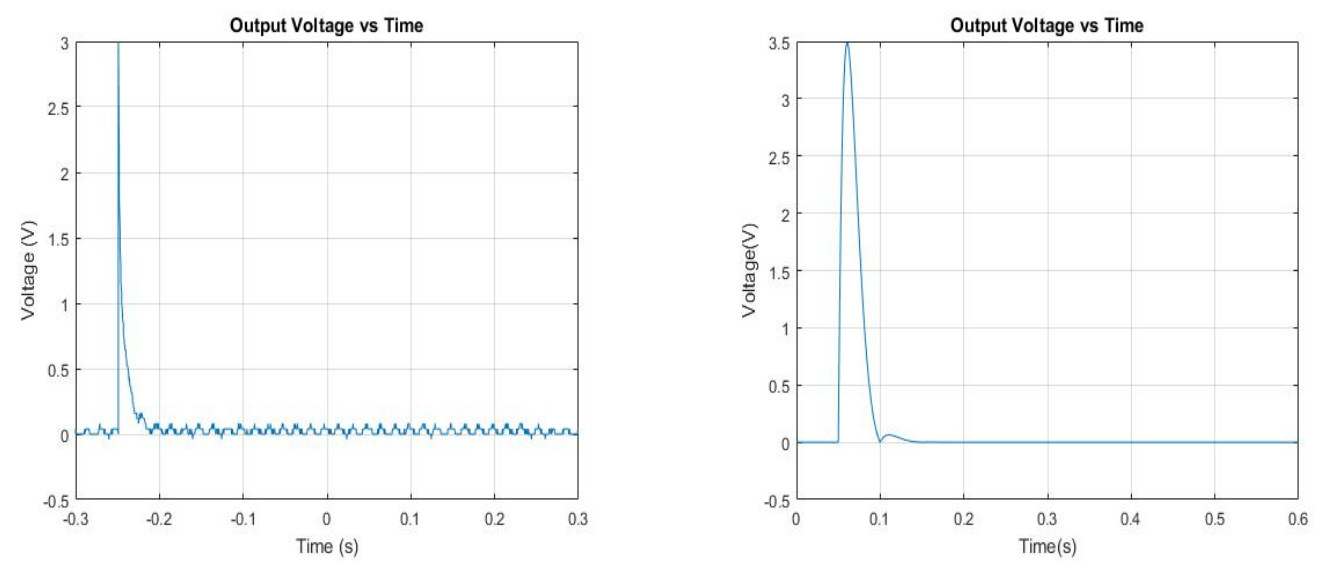

Figure 5.24: Output of the current detector with transient current for the experimental setup (left) and the simulation (right)

\subsection{Results for the vibration detector}

Figure 5.25 shows the graph of acceleration in g's (x-axis) vs. duty cycle in \% (yaxis). The accelerometer was subjected to various levels of vibration by shaking the accelerometer. The acceleration values and the duty cycle of the accelerometer output were obtained from the serial monitor of the Arduino IDE software. A graph of acceleration (g's) against the duty cycle was then plotted thereby producing a linear 
response. The slope of the graph is the sensitivity of the accelerometer which was found to be the expected $12.5 \%$ duty cycle change per g.

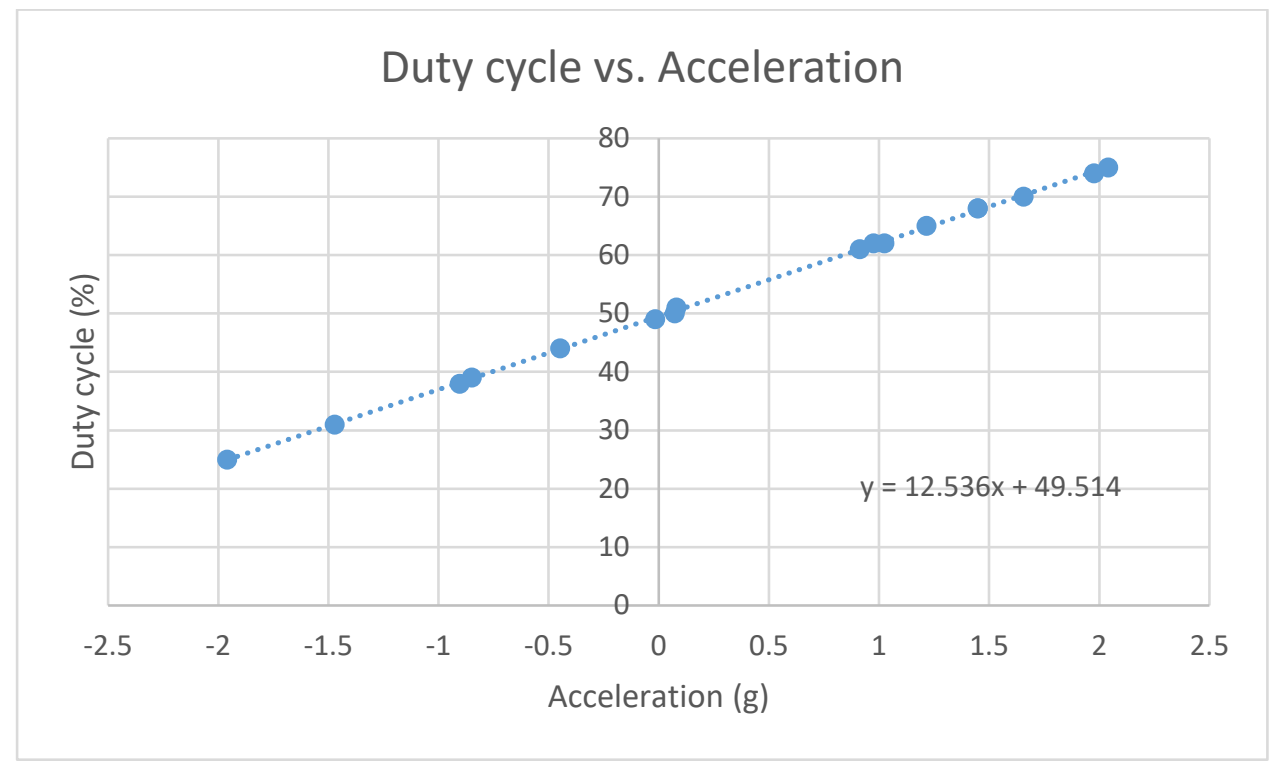

Figure 5.25: Duty cycle (y-axis) against the acceleration (x-axis)

Figure 5.26 shows the results for the vibration detector which was obtained using the serial monitor in the Arduino IDE software. The acceleration is given in milli g's. Accelerometers detect magnitude and direction of the acceleration. In the stationary or rest position, the accelerometer registers a particular value (negative acceleration of 64, in this case), due to its orientation, which changes when it experiences any kind of vibration. The prototype was tested with minor levels of vibration such as knocks. The accelerometer was shaken either sideways, to register a change in acceleration in the xaxis, or up and down, to register a change in acceleration in the y-axis. Vibration notification was given to the microcontroller whenever a significant change in either the $\mathrm{x}$-axis and/or the $\mathrm{y}$-axis was detected, as quantified by a change in acceleration in excess of the 10 milli g threshold. Any significant movement results in a change in a change of acceleration greater than 10 milli g. Thus, the 10 milli g threshold was picked as it was also suitable for avoiding false positives. 


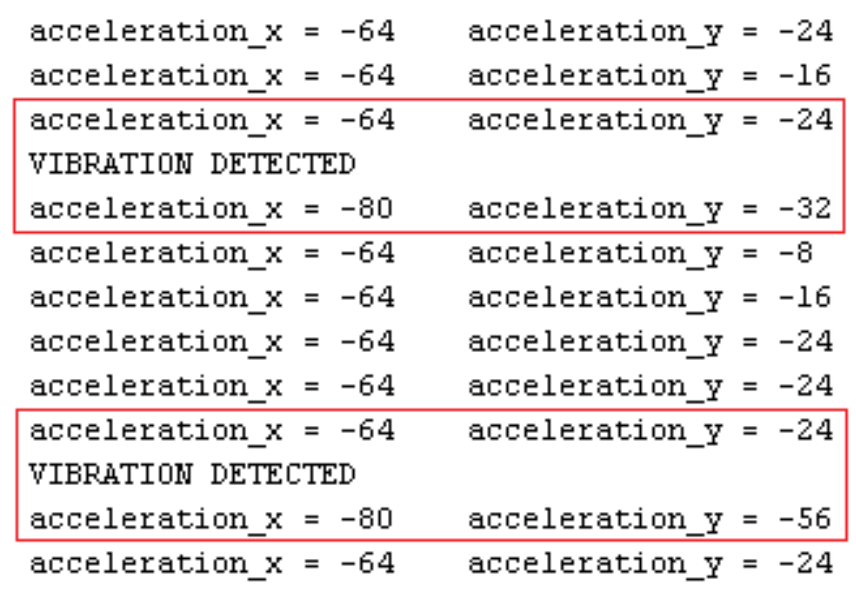

Figure 5.26: Serial monitor results for the vibration detector

5.4 Results for the combined lightning strike detector

The output voltage of the current detector and the acceleration values were fed into the microcontroller. The sampling rate is $2 \mathrm{MHz}$. The microcontroller takes 100 us to read an analog input , in this case the output voltage of the current detector. Transient currents resulting from a lighning last for more than 200 us and therefore the microcontroller is suitable for the purpose of lightning strike detection [26]. Three possible events were then created to show the response of the combined lightning strike detector. The lightning strike detector was programmed according to the flowchart given in Chapter 4. Figure 5.27 shows the vibration being detected by the vibration detector. The accelerometer was shaken without providing any input to the current detector. Since, the current detector does not produce any voltage, a lightning strike is not registered. The vibration itself is of no interest but it allows identification of the nearest tower to the lightning strike for maintenance purposes and excludes any vibrations not accompanied by a current detection since that means that the lightning strike is not to a tower or a line, hence no damage to the structure. Figure 5.28 shows the current being detected by the current detector. Input is given to the current 
detector whereas the accelerometer is not subjected to any kind of vibration. Since, the vibration detector is not registering anything, this is not the tower of impact.

Figure 5.29 shows vibration being detected by the accelerometer as well as induced voltage in the current detector. The accelerometer was shaken and at the same time, input was given to the current detector. Thus, a lightning strike detection notification is obtained as a tower which is near to the lightning strike would register both vibration as well as current readings.

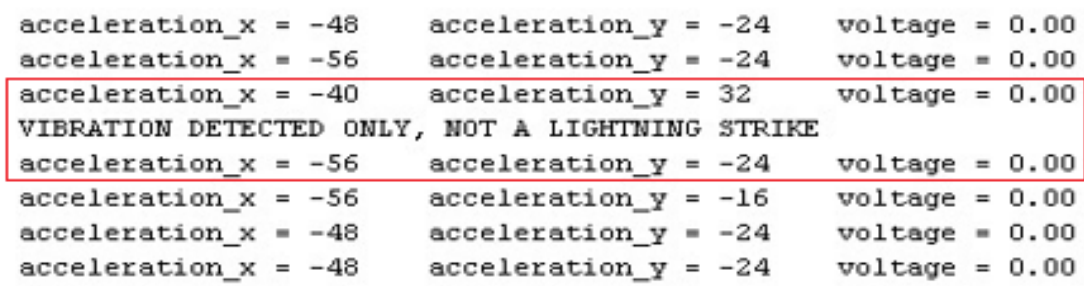

Figure 5.27: Serial monitor results for the lightning strike detector only when vibration is detected

\begin{tabular}{llll|}
\hline $\begin{array}{l}\text { acceleration_x }=-64 \\
\text { CURRENT DETECTED }\end{array}$ & acceleration_y $=-24$ & voltage $=3.42$ \\
acceleration_x $=-72$ & acceleration_y $=-24$ & voltage $=0.09$ \\
acceleration_x $=-64$ & acceleration_y $=-24$ & voltage $=0.41$ \\
acceleration_x $=-64$ & acceleration_y $=-24$ & voltage $=0.00$ \\
acceleration_x $=-72$ & acceleration_y $=-16$ & voltage $=0.00$ \\
acceleration_x $=-72$ & acceleration_y $=-24$ & voltage $=0.00$ \\
acceleration_x $=-72$ & acceleration_y $=-24$ & voltage $=0.00$
\end{tabular}

Figure 5.28: Serial monitor results for the lightning strike detector only when current is detected 


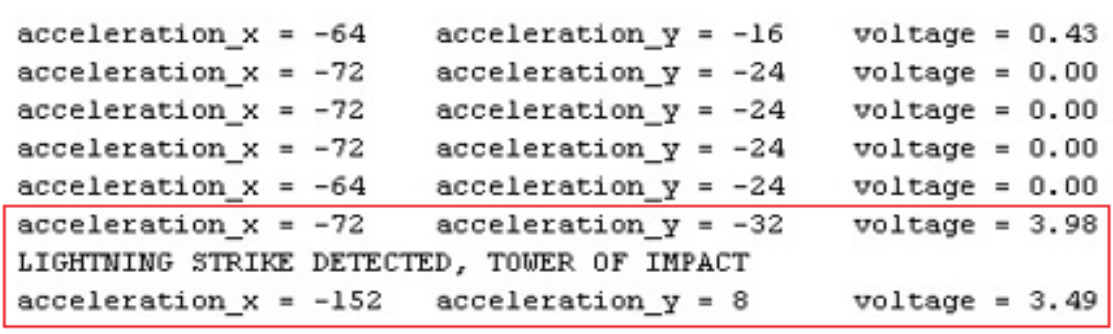

Figure 5.29: Serial monitor results for the lightning strike detector when both the current and vibration is detected

\subsection{Summary}

The results of the lightning strike detector are given in this chapter. The experimental results of the current detector conformed to the theoretical formulas and the simulation results. The vibration detector works adequately when subjected to varying levels of vibration. The combined lightning strike detector was tested using current and vibration inputs and it works as expected to provide a lightning strike detection notification when both the current as well as vibration values are significant. 


\section{CHAPTER VI}

\section{CONCLUSION AND FUTURE WORK}

\subsection{Conclusion}

Lightning strikes must be detected in a timely manner, before they lead to catastrophic consequences. This dual detection method can be used detect a lightning strike to a member of the transmission system and identify the nearest tower. The lightning strike detector, comprising the current and the vibration detector circuits, can determine the tower of the lightning impact using both current and vibration readings. If the tower registers only a current reading, then a current detection notification is given which shows that although there is a current reading, the strike occurred elsewhere. Similarly, if the tower registers only vibration reading, vibration notification is given which shows that the vibration is due to some other causes such as high wind speed or a distant strike to a tower.

A Rogowski coil was used for current detection. The transfer function of the current detector circuit was derived and used to construct the frequency response of the system to determine its response over a wide frequency range. Simulation of the circuit was then carried out in LTspice for both $100 \mathrm{kA}$ peak amplitude current and a $300 \mathrm{~mA}$ peak amplitude current. This showed that the behavior of the Rogowski coil was similar for both the cases. Due to the unavailability of instruments for large scale tests, the Rogowski coil was tested only for a small current of $300 \mathrm{~mA}$. The practical results of the current detector conformed to the theoretical formulas and simulation results. The vibration detector also worked perfectly when subjected to different levels of 
vibration. Thus, the system can be implemented for use for actual lightning strike detection in transmission towers.

\subsection{Future work}

The Rogowski coil would need to be designed with a smaller number of turns due to the high expected current, in the kA range, to turn the lightning strike detector into a commercial product that can be used in the field. As the current will be quite large, stray currents would not be enough to set off the detector. The complex conjugate of the impedance of the new Rogowski coil would then be placed across the coil. An MOV suitable for industrial applications would be used instead of the zener diodes used for the prototype. A sample and hold circuit can also be employed to ensure that the current detector output can be properly read. The lightning strike detector would also need to be tested with pulses which are of microseconds duration. The system can be expanded to involve a network of various structures with microcontrollers. The lightning strike detector would also be incorporated in a larger system with the help of TI-DSP processor, which would replace the Arduino microcontroller used in the prototype. The system would involve different sensors for other purposes, such as gunshot detection, which would be integrated using the TI-DSP for condition monitoring in transmission tower and lines. 


\section{REFERENCES}

[1] J. Bothma, "Transmission line tower collapse investigation: A case study," IEEE Power and Energy Society Conference and Exposition in Africa: Intelligent Grid Integration of Renewable Energy Resources (PowerAfrica), Johannesburg, 2012, pp. 1-8.

[2] T. Kawabata, S. Yanagawa, H. Takahashi and K. Yamamoto, "A development of a shunt lightning current measuring system using a Rogowski coil," 2013 International Symposium on Lightning Protection (XII SIPDA), Belo Horizonte, 2013, pp. 283-286.

[3] R. Y. Han et al., "Hybrid PCB Rogowski coil for measurement of nanosecondrisetime pulsed current," in IEEE Transactions on Plasma Science, vol. 43, no. 10, pp. 3555-3561, Oct. 2015.

[4] Z. Li et al, "Design of Rogowski coil with external integrator for measurement of lightning current up to 400 kA”, in Przeglad Elektrotechniczny, 87(7): 188-192, Dec. 2010.

[5] W. Yanjie, F. Changyuan, Li Yiding and W. Baoqiang, "Design of lightning location system based on photon and infrasound detection," 2007 8th International Conference on Electronic Measurement and Instruments, Xi'an, 2007, pp. 1-603-1-606.

[6] W. I. Ibrahim and Z. A. Malek, "Time-to-thunder method of lightning distance determination," 2010 IEEE International Conference on Power and Energy, Kuala Lumpur, 2010, pp. 357-362. 
[7] G. Shanqiang et al., "The recognition method of thunder based on a noiseestimation algorithm for highly non-stationary environments," 2016 33rd International Conference on Lightning Protection (ICLP), Estoril, 2016, pp. 1-5.

[8] Rongjing Cui, Xiang Cui and Zhibin Zhao, "The lightning radiation electromagnetic field data acquisition system based on virtual instrument," 2008 8th International Symposium on Antennas, Propagation and EM Theory, Kunming, 2008, pp. 11891192.

[9] W. I. Ibrahim and M. R. Ghazali, "An overview of Pekan Lightning Detection System (PLDS)," 2012 IEEE International Conference on Power and Energy (PECon), Kota Kinabalu, 2012, pp. 718-723.

[10] N. Ida, Sensors, Actuators, and Their Interfaces: A Multidisciplinary Introduction, vol. 2. IET, 2013.

[11] C. Yao, H. Wu, Y. Mi, Y. Ma, Y. Shen and L. Wang, "Finite difference time domain simulation of lightning transient electromagnetic fields on transmission lines," in IEEE Transactions on Dielectrics and Electrical Insulation, vol. 20, no. 4, pp. 1239-1246, August 2013.

[12] S. Kon and R. Horowitz, "A high-resolution MEMS piezoelectric strain sensor for structural vibration detection," in IEEE Sensors Journal, vol. 8, no. 12, pp. 2027-2035, Dec. 2008.

[13] T. Deng, D. Chen, J. Wang, J. Chen and W. He, "A MEMS based electrochemical vibration sensor for seismic motion monitoring," in Journal of Microelectromechanical Systems, vol. 23, no. 1, pp. 92-99, Feb. 2014.

[14] A. Sabato, "Pedestrian bridge vibration monitoring using a wireless MEMS accelerometer board," 2015 IEEE 19th International Conference on Computer Supported Cooperative Work in Design (CSCWD), Calabria, 2015, pp. 437-442. 
[15] “50 Hertz”, 2017 [Online]. Available: http://www.50hertz.com. [Accessed: 7July-2017].

[16] V. Skendzic and B. Hughes, "Using Rogowski coils inside protective relays," 2013 66th Annual Conference for Protective Relay Engineers, College Station, TX, 2013, pp. 1-10.

[17] M. H. Samimi, A. Mahari, M. A. Farahnakian and H. Mohseni, "The Rogowski coil principles and applications: a review," in IEEE Sensors Journal, vol. 15, no. 2, pp. 651-658, Feb. 2015.

[18] "Introduction to metal oxide varistors", 2012. [Online]. Avalable: http://www.powerguru.org/introduction-to-metal-oxide-varistors/. [Accessed: 10 Mar. 2017].

[19] D. W. Hart, Power Electronics, McGraw-Hill, NY, 2011, pp. 111-170.

[20] D. A. Ward and J. L. T. Exon, "Using Rogowski coils for transient current measurements," in Engineering Science and Education Journal, vol. 2, no. 3, pp. 105-113, June 1993.

[21] R. Han, J. Wu, Y. Jing, Q. Liu, H. Zhou and W. Ding, "Wideband Rogowski coil based on low resistance integrating resistor," 2015 IEEE International Conference on Plasma Sciences (ICOPS), Antalya, 2015, pp. 1-1.

[22] MEMSIC, “Improved, ultra low noise \pm 3 g dual axis accelerometer with digital outputs,” MXD2125 Datasheet, Mar. 2005.

[23] A. Lindsay, Smart Sensors \& Applications, Parallax Inc, 2003, pp. 65-112.

[24] “Arduino - ArduinoBoardUno", Arduino.cc, 2017. [Online]. Available: https://www.arduino.cc/en/Main/ArduinoBoardUno. [Accessed: 22- May- 2017]. 
[25] J. Takami and S. Okabe, "Observational results of lightning current on transmission towers," in IEEE Transactions on Power Delivery, vol. 22, no. 1, pp. 547-556, Jan. 2007.

[26] F. Heidler et. al, "Parameters of lightning current given in IEC 62305background, experience and outlook,” 29th International Conference on Lightning Protection, 2008, vol. 23, p. 26. 
APPENDICES 
APPENDIX A

EXPERIMENTAL SETUP

Table A.1: List of components

\begin{tabular}{|l|l|}
\hline \multicolumn{1}{|c|}{ Model Number } & \multicolumn{1}{c|}{ Description } \\
\hline Wavetek 190 & $\begin{array}{l}\text { Signal generator used for generating high frequency } \\
\text { sinusoidal current }\end{array}$ \\
\hline Aglient 34410A & Multimeter \\
\hline Aglient DSO3152A & Digital oscilloscope \\
\hline mA-2000 & Milliammeter for the measurement of input current \\
\hline MW122A & Voltage source for the generation of transient current \\
\hline
\end{tabular}




\title{
APPENDIX B
}

\section{CODE}

\author{
// accelerometer Pins \\ const int $\mathrm{xPin}=2$; // $\mathrm{X}$ pin connected to pin 2 \\ const int yPin = 3; // Y pin connected to pin 3 \\ int tolerance $=10$; // tolerance of 10 \\ int current; \\ int vibration; \\ // accelerometer limits \\ int xMin; //Minimum x Value \\ int xMax; //Maximum x Value \\ int xVal; //Current x Value \\ int yMin; //Minimum y Value \\ int yMax; //Maximum y Value \\ int yVal; //Current y Value \\ int pulseX; \\ int pulseY; \\ void setup()\{
}

//Begin Serial communication

Serial.begin(250000);

\}

void $\operatorname{loop}()\{$

// calibrate the accelerometer limits.

pulseX = pulseIn(xPin, $\mathrm{HIGH})$; 
$\mathrm{xVal}=(($ pulseX / 10) - 500) $* 8$

$\mathrm{xMax}=\mathrm{xVal}+$ tolerance;

$\mathrm{xMin}=\mathrm{xVal}$-tolerance;

pulseY = pulseIn(yPin, HIGH);

$\mathrm{yVal}=(($ pulseY / 10) - 500) * 8;

$\mathrm{yMax}=\mathrm{yVal}+$ tolerance;

$\mathrm{yMin}=\mathrm{yVal}$-tolerance;

if $((x V a l>(x M a x)) \|(x V a l<(x M i n)))\{$

vibration $=1 ;\}$

if $((y V a l>(y M a x)) \|(y V a l<(y M i n)))\{$

vibration $=1 ;\}$

// print the acceleration

Serial.print ("acceleration_x = ");

Serial.print(xVal);

// print a tab character:

Serial.print("\t");

Serial.print("acceleration_y = ");

Serial.print(yVal);

Serial.print("\t");

// read the input on analog pin 0 :

int sensorValue $=$ analogRead $(\mathrm{A} 0)$;

// Convert the analog reading (which goes from 0 - 1023) to a voltage $(0-5 \mathrm{~V})$ :

float voltage $=$ sensorValue $*(5.0 / 1023.0)$;

// print out the value you read:

Serial.print("voltage = ");

Serial.print(voltage);

Serial.println(); 
if (voltage $>0.5)\{$

current $=1 ;\}$

if $(($ vibration $==1) \& \&($ current $==1))\{$

Serial.print("LIGHTNING STRIKE DETECTED, TOWER OF IMPACT");

Serial.println();

\} else if $(($ vibration $==1) \& \&($ current $==0))\{$

Serial.print("VIBRATION DETECTED ONLY, NOT A lightning STRIKE");

Serial.println();

\} else if $(($ vibration $==0) \& \&($ current $==1))\{$

Serial.print("CURRENT DETECTED");

Serial.println(); \}

vibration $=0$;

current $=0$; 